\title{
Context-aware system for pre-triggering irreversible vehicle safety actuators
}

\author{
Dennis Böhmländer ${ }^{\mathrm{a}, \mathrm{b}, *}$, Tobias Dirndorfer $^{\mathrm{a}}$, Ali H. Al-Bayatti ${ }^{\mathrm{b}}$, Thomas Brandmeier ${ }^{\mathrm{c}}$ \\ a AUDI AG, 85045 Ingolstadt, Germany \\ ${ }^{\mathrm{b}}$ De Montfort University, Leicester, United Kingdom \\ c CARISSMA, Technische Hochschule Ingolstadt, Germany
}

\begin{abstract}
A B S T R A C T
New vehicle safety systems have led to a steady improvement of road safety and a reduction in the risk of suffering a major injury in vehicle accidents. A huge leap forward in the development of new vehicle safety systems are actuators that have to be activated irreversibly shortly before a collision in order to mitigate accident consequences. The triggering decision has to be based on measurements of exteroceptive sensors currently used in driver assistance systems. This paper focuses on developing a novel context-aware system designed to detect potential collisions and to trigger safety actuators even before an accident occurs. In this context, the analysis examines the information that can be collected from exteroceptive sensors (precrash data) to predict a certain collision and its severity to decide whether a triggering is entitled or not. A five-layer context-aware architecture is presented, that is able to collect contextual information about the vehicle environment and the actual driving state using different sensors, to perform reasoning about potential collisions, and to trigger safety functions upon that information. Accident analysis is used in a data model to represent uncertain knowledge and to perform reasoning. A simulation concept based on real accident data is introduced to evaluate the presented system concept.
\end{abstract}

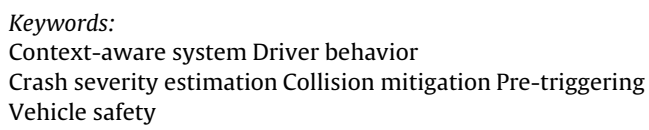

\section{Introduction}

It is estimated that on the world's roads $20-50$ million people suffer injuries each year by traffic collisions. This trend will worsen according to World Health Organization (WHO) predictions so that by 2030 , traffic fatalities will be the fifth most common cause of death in the world (World Health Organization, 2013). Unfortunately, most accidents are caused by human error, and the reasons are typically related to incorrect judgment (e.g. distance to preceding vehicle) or lack of driver attention (Bayless et al., 2012). The primary goals of the vehicle safety research of today, therefore, is the complete avoidance of traffic accidents. Failing this, a secondary goal should be the optimal protection of the vehicle passengers and Vulnerable Road Users (VRUs) (i.e. pedestrians). In this respect, the automobiles sense of 'sight' and its exteroceptive sensors, can play an increasingly important role. Intelligent Vehicles (IV) are able to perceive and model their environment and correctly recognize potential dangers. Today, a special focus is on the investigation of methods to activate pre-crash triggered safety applications in

\footnotetext{
* Corresponding author at: AUDI AG, 85045 Ingolstadt, Germany. E-mail address: dennis.boehmlaender@audi.de (D. Böhmländer).
}

order to increase the vehicle crash performance and the passenger protection.

Research activities in the past years show benefits of new occupant restraint systems which are deployed immediately prior to a collision. Contemporary passenger safety systems combine air bags and three-point-automatic seat-belts to reduce the passengers risk of injury. For a proper generation of the triggering decision, vehicle dynamics, peripheral intrusion and occupants positions are measured and evaluated (Feser et al., 2006; Chan, 2000; Rölleke and Köhler, 2001). In comparison, novel systems which have to be triggered shortly before a vehicle crash can provide a larger cushion volume. In special load cases occupant loads can be reduced by up to $20 \%$ on average (Wohllebe et al., 2006; Gstrein and Sinz, 2009). This will offer occupants new interior concepts and profoundly changed sitting positions. External airbags and structure-airbag concepts (i.e. synthetic pressure hoses which are filled with gas before an impact) can extend the vehicle's crumple zones to provide additional energy absorption of impacts (Hakki and Hakki, 2008; Chung et al., 2014; Löffler and Quedenbaum, 2014; Unselt et al., 2015). The introduction of pre-crash measures not only makes sense for occupant protection, but is also an important element in upcoming actuator technology to protect VRUs (Mishra, 2012; Choi and Kim, 2010). Fig. 1 outlines the chronological sequence of 


\begin{tabular}{|c|c|}
\hline \multicolumn{2}{|c|}{ Abbreviations } \\
\hline$c$ & stiffness \\
\hline$m$ & mass \\
\hline$J_{z}$ & momentum around $z$-axis \\
\hline $\mathbf{L}$ & angular momentum \\
\hline I & Impulse \\
\hline$\tau$ & trajectory \\
\hline $\mathcal{R}$ & risk \\
\hline $\mathbf{x}$ & state vector \\
\hline $\mathbf{r}, x, y$ & distance \\
\hline$v, \dot{x}$ & velocity \\
\hline$a, \ddot{x}$ & $\begin{array}{l}\text { acceleration } \\
\text { heading angle }\end{array}$ \\
\hline$\dot{\psi}$ & yaw rate \\
\hline $\boldsymbol{\omega}(t)$ & angular velocity \\
\hline$\Delta V$ & change in velocity \\
\hline OLC & occupant load criterion \\
\hline Cs & crash severity \\
\hline$k$ & restitution coefficient \\
\hline IP & impact point \\
\hline$\alpha$ & impact angle \\
\hline$C G$ & center of gravity \\
\hline$d$ & $y$-distance between IP and CG \\
\hline$l$ & length \\
\hline$w$ & width \\
\hline $\mathbf{S}$ & crash constellation \\
\hline$O L$ & collision overlap \\
\hline$\mu$ & expected value \\
\hline TS & triggering strategy \\
\hline$N F$ & no fire \\
\hline$C F$ & can fire \\
\hline$M F$ & must fire \\
\hline TTC & time to collision \\
\hline$t_{\text {algo }}$ & computing time \\
\hline$T_{S}$ & sample time \\
\hline$t_{F}$ & triggering time \\
\hline$t_{l}$ & system latency \\
\hline $\mathrm{F}_{\beta}$ & weighted average of precision and recall \\
\hline$\sigma$ & variance \\
\hline $\mathbf{R}$ & noise covariance matrix \\
\hline $\mathbf{P}$ & a posteriori error covariance matrix \\
\hline$f$ & probability density function \\
\hline
\end{tabular}

a vehicle collision and demonstrates the basic idea to pre-trigger irreversible vehicle safety actuators. State-of-the-art systems measure and assess the vehicle's acceleration and structure-borne sound to make a safe triggering decision. Compared to today's restraint triggering, the proposed approach in this paper only uses exteroceptive sensor observations to detect unavoidable collisions and to assess the crash severity in order to trigger restraint systems shortly before collision. This paper discusses requirements and a system concept to activate vehicle safety systems just before an unavoidable collision and is structured as follows: Section 2 addresses related work and identifies the central challenges. Section 3 introduces a context-aware system for pre-triggering and discusses possible methods to predict a collision as well as defines requirements. Section 4 addresses crash severity and vehicle accident analysis. In Section 5 a model is presented to predict the crash severity and to trigger safety actuators. The evaluation of the described methodology based on real accident data is presented in Section 6. Finally, in Section 7 conclusions are drawn and the further research direction is described.

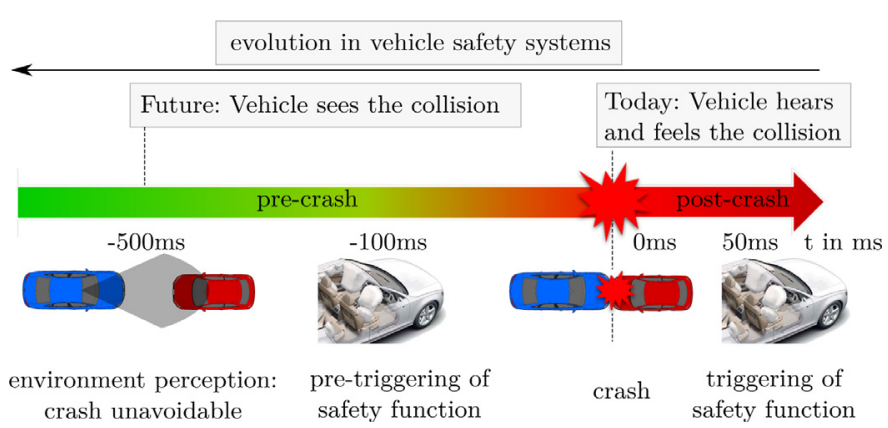

Fig. 1. Basic concept for pre-triggering irreversible vehicle safety actuators.

\section{Related work}

This section puts this work into the context of relevant literature. Active restraint systems like air-bags and seat-belts are triggered to reduce the vehicle occupants risk of injury when an accident occurs. To cushion the occupant motion best during a vehicle accident, airbags have to be triggered and inflated extremely rapidly after the collision occurs. Beyond that it is not only a decision if or not an activation is necessary but also which actuator and at what time it has to be triggered. A typical state-of-the-art sensing concept consists of pressure sensors in the vehicle passenger doors to detect side collisions and acceleration sensors in all spatial directions at the transmission tunnel (near to the vehicles center of gravity) as well as two acceleration sensors in the vehicle front.

Different studies show that triggering of safety actuators (i.e. smart air-bags) shortly before a collision can significantly reduce the injury risk and severity of the vehicle occupant. In order to predict a certain crash severity, information from the vehicle environment has to be collected and analyzed.

Mühlfeld et al. (2013) present an approach to trigger driveradaptive pre-crash systems via statistical behavior modeling with the focus on system performance and user acceptance. The proposed methodology analyses past situations to calculate a driver individual activation threshold. The presented approach estimates the criticality of an oncoming collision for pre-crash triggering, but does not take into account a possible crash severity. Cho et al. combines exteroceptive sensor and longitudinal accelerometer measurements to improve the integrity of airbag deployment decisions (Kwanghyun Cho, 2011). The presented approach only uses Radar sensor measurements as an additional input where the deployment decision is mainly based on the in-crash deceleration measurement.

A methodology to estimate optimal trigger times of an adaptive frontal restraint system for an oncoming collision can be found in (Wallner et al., 2009; Eichberger et al., 2009). An algorithm is presented, which calculates force levels with acquired parameters by combining three separate models (vehicle model, collision model and occupant model). The collision and occupant model uses a spring mass system to calculate the maximum and mean acceleration of the occupant. As input parameters, the models use the collision speed, mass and stiffness of the colliding vehicle. The investigation considers straight frontal collisions with full overlap and determine the stiffness according to different sets of mass categories. However, it is not described how the mass and collision speed can be acquired or predicted.

Rao et al. proposes an architecture consisting of a vision system and a Radar or Lidar sensor to trigger external airbags (Rao et al., 2005). Measurements from a Radar and Lidar sensor are used to determine the relative velocity and distance to the potential collision opponent. This information is then fused with the collision opponents classification (it can distinguish between pedestrians or vehicles) provided by the vision system in order to deploy the 
external airbags at the right activation level. However, they do not predict any crash severity and only use the measured relative velocity as a criterion. King focuses in (King, 2005) on a similar architecture but also determines a potential crash severity. They define the crash severity as the difference between the kinetic energies or momentums of the ego vehicle and its collision opponent. For the crash opponent's kinetic energy he estimates the mass only based on its class. This very rudimentary crash severity calculation does not take any crash mechanics into account and can be imprecise in some crash constellations.

Browne et al. (2012) present an architecture to deploy occupant safety devices using fused measurements of an exteroceptive sensor and vehicle communication. This approach includes an exchange of vehicle condition-defining signals when the probability of a potential crash is greater than a threshold value and a crash severity prediction between the vehicles. In this case, the possible collision opponent is intended to provide information such as vehicle dynamics (i.e. speed), geographic position data from Global Navigation Satellite System (GNSS) receiver and also crash relevant parameters (e.g. mass, dimensions and stiffness).

Andreas Meier (2014) describe an approach to generate a deployment decision based on a learned prediction model that is able to identify to which of a set of seven crash severity categories an impending collision scenario belongs to. He uses velocities, collision angles and impact points at the vehicle fronts estimated by pre-crash sensors as an input for his classification algorithm. Different classifiers and their performances are presented for a vehicle-to-vehicle (V2V) collision simulation data set generated by finite element method (FEM). The REPTree, a fast decision tree learner, performs best on simulation data with a classification performance of $84 \%$. It is not specified in detail how the input parameters can be determined and in which precision they are needed to make safe decisions. Further it is still unknown if a knowledge based system (artificial intelligence) trained on simulation results alone would be accepted to make high safety critical decisions.

Summarizing it can be said that there are different physical models to predict an imminent collision using forward-looking sensors. Also physical or data based models exist to predict a potential crash severity for specific crash scenarios (e.g. full overlap straight head on V2V collisions). Also the combination of forward-looking and in-crash sensor measurements, to make a more precise decision is shown. However, it is not presented how a system can accurately trigger safety actuators prior to a collision. So far it has not been determined what system requirements have to be met to deal with uncertainties in sensor measurements and complex traffic situations in order to perform reasoning about the potential collision. The mere fact that such a system does not exists in today's volume production vehicles demonstrates the need of investigation in this area. Accordingly, this paper addresses this question: which quantities have to be measured prior an impending collision to reason about its severity and to make it feasible to sufficiently interpret the triggering decision?

\section{Methodology and system design for pre-crash triggering}

In the following section a system architecture and the reasoning methodology for pre-crash triggering is presented. The system must respond quickly to unknown situations based on environment information to assess the threat to trigger safety functions. This means that the system should not only react to sensor measurements, but must interpret the current situation. The main requirement is to deal with situations in which non-unique or where not enough input parameters of sensors are available to carry out a complete situation assessment. At this point, context

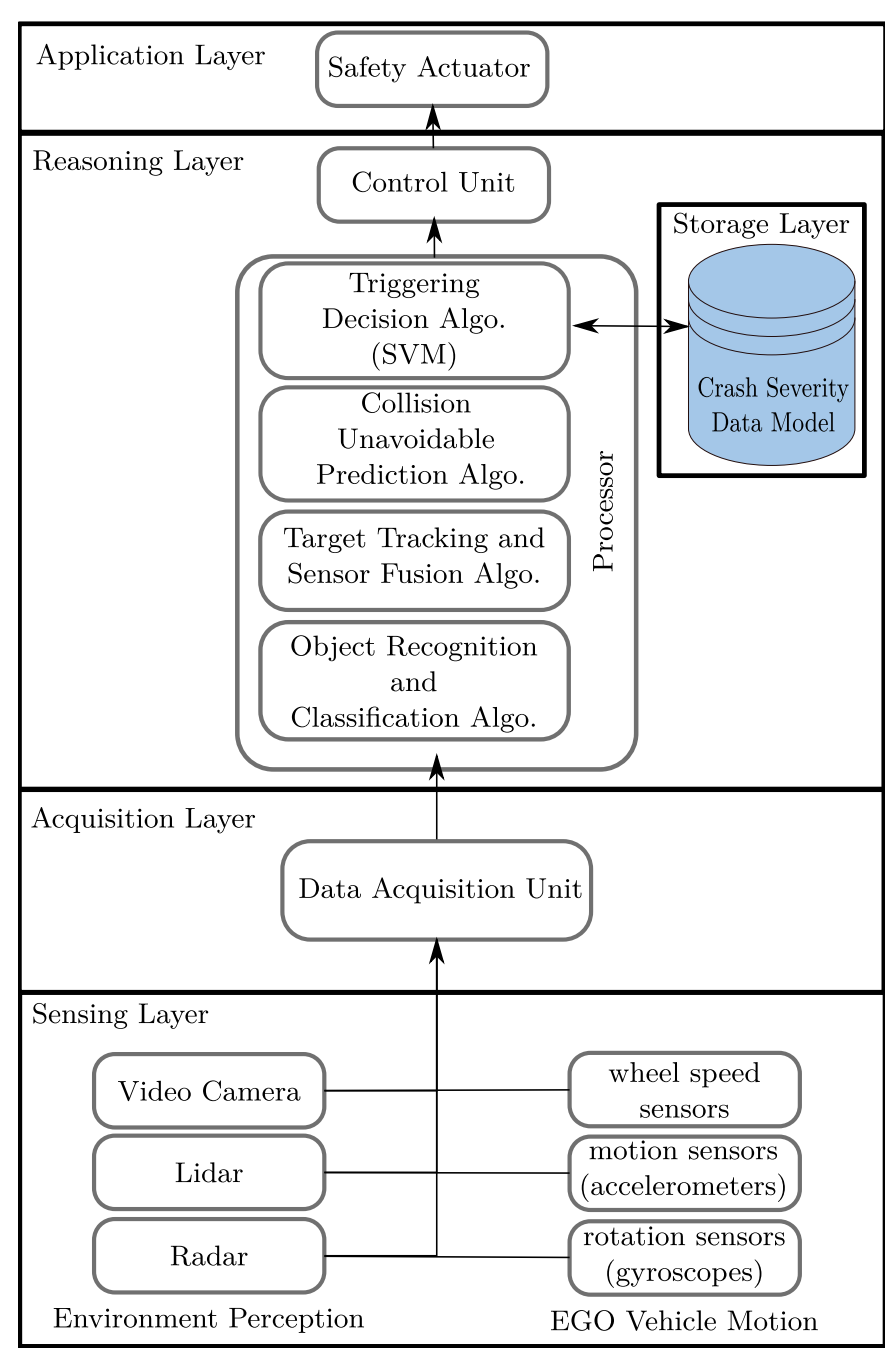

Fig. 2. System architecture for triggering safety actuators based on environment perception.

information is used to trigger safety functions. Therefore, the system's methodology is based on low level context (raw sensor data) and high level context (pre-crash situation) to predict a collision and its crash severity to derive triggering decisions. In order to reduce complexity, the work focuses on V2V collisions. V2V accidents are the most probable scenarios in Germany to suffer severe or fatal injuries. Especially head on frontal collisions with another oncoming vehicle comprise $21 \%$ of all fatal accidents in 2012 (Statistisches Bundesamt, 2012). The presented contextaware system is divided into five layers: sensing layer, acquisition layer, reasoning layer, storage layer and application layer. Fig. 2 shows the proposed context-aware system with the basic steps that have to be performed to derive a pre-triggering decision (activation of safety actuators before collision). The sensing layer comprises the environmental and the ego vehicle motion sensors. The layer includes a video camera, a Lidar and Radar sensor for environmental perception as well as wheel speed sensors, accelerometers and gyroscopes for ego vehicle motion sensing. The primary task of the exteroceptive sensor set is the detection and classification of objects in the vehicle environment. For a collision prediction it has to be determined what kind of object and where it is located relative (distance) to the ego vehicle and how it moves (velocity and heading). Of particular interest is which class can be assigned to the object (e.g. small vehicle, biker, tree, truck, etc.) to identify it and to derive more information. The classification of objects is a classic 
application of video camera systems. Video camera measurements have limited accuracy in the object position. Radars can measure directly velocity, the object position and have a good sample rate but cannot provide the object's appearance and shape. Lidars can measure very accurate distances and the object shape (by the contour), but a sufficient number of layers are required to provide the object's appearance. A complementary fusion of these sensors resolves the incompleteness of sensor data and provides redundancy to improve the safety integrity. The heterogeneous raw sensor data (low level context) is collected, analyzed and associated in the data acquisition layer. The reasoning layer includes sensor fusion, classification and target tracking based on the low level context. Necessary sensor data association, object recognition and classification algorithms will not be discussed at this point and it is refereed to our previous work (Böhmländer et al., 2014). The reasoning layer uses then high level context to characterize the pre-crash situation: if a collision is regarded as unavoidable the decision is made by applying a Crash Severity Data Model (CSDM). The CSDM is depicted in the storage layer. The application layer represents possible safety actuators that can be triggered in the pre-crash phase (i.e. occupant airbag).

\subsection{Target tracking and sensor fusion}

The sensor data fusion layer uses a state of the art 2D target tracking and fusion architecture. In order to predict possible collisions in the near future the potential crash opponent's current position and kinematic has to be determined using the ego vehicle's exteroceptive sensor measurements. First of all, the change of position of the ego vehicle has to be estimated using kinematic measurements. For estimating the ego vehicle dynamics a constant turn rate and acceleration (CTRA) model is used Rong Li and Jilkov (2003). The CTRA is a coordinate-coupled two dimensional horizontal motion model that provides satisfactory accuracy for proof of concept. In contrast to the single-track model the CTRA does not need vehicle specific parameters (i.d. lateral tire stiffness). The single-track model represents more accurately the dynamics and considers the effects of the tire forces (Schofield, 2008). By measuring the ego vehicle motion, its state vector can be written in global Cartesian coordinate system $S_{g}$ with the origin $O_{g}$ written as:

$\mathbf{x}_{n}^{E}=\left[\begin{array}{lllll}x_{n}^{O_{g}} & y_{n}^{O_{g}} & O_{n} & \dot{\psi}_{n}^{O_{g}} & v_{x, n}^{O_{g}}\end{array}\right]^{T}$,

where $x_{n}^{O_{g}}$ and $y_{n}^{O_{g}}$ are the Cartesian coordinates of the ego vehicle's center of gravity $C G, \psi_{n}^{O g}$ is the heading angle, $\dot{\psi}_{n}^{O_{g}}$ is the yaw rate and $v_{x, n}^{O g}$ is respectively the longitudinal velocity at the time $n$ with the sample time $T$ between time steps. The $i$-th object state vector at the time step $n$ can be expressed in the ego vehicle's Cartesian coordinates as follows:

$\mathbf{x}_{n}^{O_{i}}=\left[\begin{array}{llll}r_{x, n}^{S^{\prime}} & v_{x, n}^{O_{g}, S^{\prime}} & r_{y, n}^{S^{\prime}} & v_{y, n}^{O_{g}, S^{\prime}}\end{array}\right]^{T}$.

With fused data from ego vehicle's predictive sensor array, it is possible to estimate the object's state vector and its covariance matrix as well as its dimensions and type. Since the ego vehicle is moving, a body fixed Cartesian coordinate system with it is origin in the ego vehicle's CG is used for object tracking. The object position $\mathbf{r}^{O}=\left[\begin{array}{ll}r_{x, n}^{S^{\prime}} & r_{y, n}^{S^{\prime}}\end{array}\right]^{T}$ is notated relative to the ego coordinate system origin $S^{\prime}$ at the time step $n$. Altendorfer demonstrates in Altendorfer (2009) that using velocity which is estimated over ground shows best results for tracking. Therefore object's velocity $\mathbf{v}_{n}^{O_{g}, S^{\prime}}$ over ground measurement is used and is rotated into the ego coordinate system $S^{\prime}$.

In addition to the dynamics, the object's type (i.e. vehicle, pedestrian) has to be defined by the class and the body dimensions. These are for example the width $w^{O}$ and length $l^{O}$. In the proposed system layer, the object type will be classified by analyzing video frames in real-time (Böhmländer et al., 2014). An extended Kalman filter (EKF) is used to predict the state vectors based on measurements observed over time. The state of the EKF is represented by two variables: $\hat{\mathbf{x}}_{n \mid n}$, the a posteriori state estimate at time $n$ given observations up to and including at time $n$; and $\hat{\mathbf{P}}_{n \mid n}$, the a posteriori error covariance matrix (a measure of the estimated accuracy of the state estimate). For a detailed derivation of the equations used in the EKF the reader is refereed to Dirndorfer et al. (2011). The approach is similar but differs considerably in the underlying dynamic system model. In this work a constant acceleration (CA) model is applied. This model uses the measured object velocity as an additional input and models the acceleration. For object state estimation and tracking a modified State Vector Fusion (SVF), a major sensor fusion model for good velocity estimates, is used (Naidu and Gopalratnam, 2010). This SVF architecture first estimates the state vectors for Radar $\mathbf{x}_{n \mid n}^{r}$ and Lidar $\mathbf{x}_{n \mid n}^{l}$ separately using two independent EKF. The Radar velocity measurement is used as an additional input for the Lidar tracker. In Cartesian coordinates the measurement vectors can be written as follows (to make it more comprehensible hereinafter the coordinates system notation will be omitted): $\mathbf{z}_{n}^{r}=$ $\left[\begin{array}{llll}r_{x, n}^{r} & v_{x, n}^{r} & r_{y, n}^{r} & v_{y, n}^{r}\end{array}\right]^{T}$ and $\mathbf{z}_{n}^{l}=\left[\begin{array}{llll}r_{x, n}^{l} & v_{x, n}^{r} & r_{y, n}^{l} & v_{y, n}^{r}\end{array}\right]^{T}$, and the noise covariance matrices as: $\mathbf{R}^{r}=\operatorname{diag}\left(\sigma_{r_{x}^{r}}^{2}, \sigma_{v_{x}^{r}}^{2}, \sigma_{r_{y}^{r}}^{2}, \sigma_{v_{y}^{r}}^{2}\right)$ and $\mathbf{R}^{l}=\operatorname{diag}\left(\sigma_{r_{x}^{l}}^{2}, \sigma_{v_{x}^{r}}^{2}, \sigma_{r_{y}^{l}}^{2}, \sigma_{v_{y}^{r}}^{2}\right)$. Afterwards the resulting two state vectors are fused by evaluating the estimated state error covariances of each track:

$$
\begin{aligned}
\hat{\mathbf{x}}_{n \mid n}^{f} & =\hat{\mathbf{x}}_{n \mid n}^{r} \hat{\mathbf{P}}_{n \mid n}^{r}\left[\hat{\mathbf{P}}_{n \mid n}^{r}+\hat{\mathbf{P}}_{n \mid n}^{l}\right]^{-1}\left(\hat{\mathbf{x}}_{n \mid n}^{l}-\hat{\mathbf{x}}_{n \mid n}^{r}\right), \\
\hat{\mathbf{P}}_{n \mid n}^{f} & =\hat{\mathbf{P}}_{n \mid n}^{r} \hat{\mathbf{P}}_{n \mid n}^{r}\left[\hat{\mathbf{P}}_{n \mid n}^{r}+\hat{\mathbf{P}}_{n \mid n}^{l}\right]^{-1} \hat{\mathbf{P}}_{n \mid n}^{r},
\end{aligned}
$$

whereby $\hat{\mathbf{x}}_{n \mid n}^{f}$ is the fused a posteriori state estimate and $\hat{\mathbf{P}}_{n \mid n}^{r}$ the fused a posteriori error covariance matrix.

\subsection{Collision prediction and threat assessment}

Unavoidable collisions can be detected by predicting the future motion of the ego vehicle and other road users (Lefévre et al., 2014). With respect to the actual estimated ego and object state, driving dynamic limits and the driver behavior possible trajectories $\tau_{j}$ with $j \in \mathbb{N}$ can be predicted for a short-term. Statistical sampling can be applied to calculate a large number of probable ego vehicle and its surrounded objects trajectories by diversifying the accelerations $a_{\mathrm{x}}$ and $a_{\mathrm{y}}$ in longitudinal and lateral direction. Threat assessment is the process of analyzing the actual driving situation. Possible dangers are evaluated and it can be decided whether a safety actuator has to be triggered. Eidehall and Petersson (2008) and Jansson (2005) distinguish between deterministic and statistical threat assessment for the use in vehicle safety. Statistical threat assessment, in comparison to deterministic approaches, deals with uncertainty in the decision process. The proposed decision processes have in common that they evaluate possible collisions to determine if the crash is unavoidable or to find the best evasion trajectory (Eidehall and Petersson, 2008; Althoff et al., 2009). However, both decision approaches do not take into account the possible crash severity. For pre-triggering, the threat can be regarded as a measure of risk. It is formally defined as the combination of the probability and the crash severity $C s$ of an impending crash. Mathematically, the risk $\mathcal{R}$ can be expressed as a tuple:

$\mathcal{R}=\left\{\left\langle\left(\tau_{i}^{(E)}, \tau_{j}^{(O)}\right), f\left(\tau_{i}^{(E)}, \tau_{j}^{(O)}, C s_{i j}\right)\right\rangle\right\}$,

whereby $\left(\tau_{i}^{(E)}, \tau_{j}^{(O)}\right)$ is the collision scenario description defined by the possible future trajectories $\tau_{i}^{(E)}$ and $\tau_{j}^{(O)}$, the probability $f$ of that 
scenario and the crash severity $C s_{i j}$ of that scenario. To determine $\mathcal{R}$, the probability of the trajectories selected by the ego driver $\tau_{i}^{(E)}$ and object driver $\tau_{j}^{(O)}$ as well as the possible $C s_{i j}$ and its probability must be determined. The crash severity $C s$ is regarded as a random variable. In the following chapter accident data and crash severity models are presented and analyzed to be able to provide statements about $C S$ and its probability density function $f_{C S}$.

\section{Crash severity and accident analysis}

In the following, models and important parameters are analyzed to predict crash severity using accident data.

\subsection{Occupant injury criteria and crash severity models}

The crash pulse is a typical feature of the vehicle structural response in an accident. It is an acceleration time histories series which defines the collision event at a certain location in the vehicle and an important characterization to describe a vehicle collision. The crash pulse shows a strong dependency on vehicle types and collision speeds. By analyzing the crash pulse it can be distinguished between different crash types (e.g. soft and hard crash). Further, the crash pulse characteristics during the collision are an important measure of crash severity. In crash worthiness studies and occupant restraint deployment algorithms basic kinematic variables are used. These basic criteria are associated with the crash pulse, for example, the maximal acceleration $a_{\max }$, the point in time when the vehicle velocity is zero $t_{v}=0$ or the sliding mean $S_{25}(25 \mathrm{~ms}$ integration window size for calculating the average deceleration) (Kübler et al., 2008; Huang, 2002; Chan, 2002; Varat, 2003). These measures are related to the vehicle kinematic response during the crash and can be used to judge the crash severity (Huang, 2002). Looking in detail for a scalar variable to best measure crash severity, the change in velocity $(\Delta V)$ is very suitable. The $\Delta V$ expresses the energy that must be imbibed by the vehicle structure and indicates the loading on the occupants. According to O'Brien (O'Brien, 2010) $\Delta V$ is an excellent choice to measure crash severity in V2V collisions since it takes into account all differences in the initial momentum, leftover velocities and the rebound of the two colliding vehicles. With the help of easy mathematical operations, all of the mentioned crash severity criteria can be deduced from the crash pulse. In comparison, the Occupant Load Criterion $(O L C)$ and Frontal Crash Criterion (FCC) are based on simplified mechanical models. The $O L C$ rates the occupant displacement and decelerations by modeling the restraint forces which act on the vehicle occupant chest (Kübler et al., 2008). This mechanical model takes an optimal restraint of the vehicle occupant into account and approximates a constant deceleration of this occupant over a relative path of $235 \mathrm{~mm}$ to the vehicle. The required occupant deceleration on this path defines the OLC. A detailed description of the OLC, FCC and the advanced criterion $\mathrm{OLC}++$ can be found in (Kübler et al., 2009; Lefer and Rebolloso, 2012). Previous research (O’Brien, 2010; Metzger et al., 2010) show that biomechanical injury severity, here, for example, the Maximum Abbreviated Injury Scale (MAIS) (Thomas et al., 2008; Schmitt et al., 2007), and the mechanical crash severity are very well correlated. Simplified models are needed to predict the crash severity prior to an imminent collision. Crash pulses can be modeled using classical mechanics: Multi-body systems and the principles of impulse and momentum.

\subsubsection{Multi-body (MB) crash model}

Schöneburg presents in Schöneburg (1988) a multi-body (MB) system model consisting of multiple masses and non-linear force-displacement characteristics to model the vehicle front and occupants dynamic behavior in frontal crash load cases. Also Huang proposes in Huang (2002) a one dimensional crash model with more than two masses and any number of non-linear energy absorbers to predict the impact dynamics. The crash pulse $\mathrm{x}(t)$ can be predicted by solving the following differential equation in matrix form:

$\mathbf{M x}(t)+\mathbf{D} \dot{\mathbf{x}}(t)+\mathbf{C} \mathbf{x}(t)=\mathbf{f}(t)$,

with the mass matrix $\mathbf{M}$, the damping matrix $\mathbf{D}$, stiffness matrix C and external forces (load) vector $\mathbf{f}(t)$. In order to solve Eq. (5) numerical, it can be reduced to a first-order differential equation as follows:

$$
\begin{aligned}
\dot{\mathrm{z}}(t)= & {\left[\begin{array}{l}
\mathbf{x}(t) \\
\dot{\mathrm{x}}(t)
\end{array}\right]=\left[\begin{array}{l}
\mathbf{a}(t) \\
\mathbf{v}(t)
\end{array}\right]=\mathbf{A z}(t)+\mathbf{u}=\left[\begin{array}{ll}
0 & \mathbf{I} \\
-\mathbf{M}^{-1} \mathbf{C} & -\mathbf{M}^{-1} \mathbf{D}
\end{array}\right]\left[\begin{array}{l}
\dot{\mathrm{x}} \\
\mathbf{x}
\end{array}\right] } \\
& +\left[\begin{array}{l}
0 \\
-\mathbf{M}^{-1} \mathbf{f}
\end{array}\right] .
\end{aligned}
$$

The model parameters ( $\mathbf{M}, \mathbf{D}$ and $\mathbf{C}$ ) can be derived for each vehicle from measured behavior in standard crash tests and computer simulations (finite element analysis). The load vector $\mathbf{f}$ depends on the kinetic energy. The presented model above can provide good results in reconstruction of vehicle collisions against a rigid wall, however, to predict a V2V collision the information about the vehicle's crash opponent has to be known in detail. It is difficult to obtain these parameters by a forward looking sensor array. In order to reduce the input parameters that have to be estimated, simplified MB models can be used. In full frontal V2V collisions, the ego vehicle crash pulse can be roughly estimated by solving a mass-spring-system consisting of only two masses and two springs (Wallner et al., 2009). In (Huibers and De Beer, 2001) the frontal vehicle stiffness of a number of different vehicle classes are analyzed with the outcome that the load distribution shows a dominant influence of the engine contact. Thus, to model the crash outcome more precisely, the usage of a MB crash model consisting of four springs and four masses is more preferable. In this case, the MB model considers the vehicle engine masses $m_{\text {eng }}^{E}$ and $m_{\text {eng }}^{O}$ as well as the initial vehicle front stiffness (between bumper and engine) $c_{\text {init }}^{E}, c_{\text {init }}^{O}$ and secondary vehicle front stiffness (between engine and the passenger compartment) $c_{\mathrm{sec}}^{E}$ and $c_{\mathrm{sec}}^{O}$. To estimate the crash pulse, Eq. (6) can be solved using the resulting stiffness matrix:

$\mathbf{C}=\left[\begin{array}{llll}c_{\mathrm{sec}}^{E} & -c_{\mathrm{sec}}^{E} & 0 & 0 \\ -c_{\mathrm{sec}}^{E} & c_{\mathrm{sec}}^{E}+c_{r e s} & c_{\text {res }} & 0 \\ 0 & -c_{\text {res }} & c_{\mathrm{sec}}^{O}+c_{\text {res }} & -c_{\mathrm{sec}}^{O} \\ 0 & 0 & -c_{\mathrm{sec}}^{O} & c_{\mathrm{sec}}^{O}\end{array}\right]$,

with:

$c_{\text {res }}=\frac{c_{\text {init }}^{E} c_{\text {init }}^{O}}{c_{\text {init }}^{E}+c_{\text {init }}^{O}}$,

damping matrix $\mathbf{D}=0_{4 \times 4}$ and mass matrix $\mathbf{M}=$ $\left[m_{\text {comp }}^{E}, m_{\text {eng }}^{E}, m_{\text {eng }}^{O}, m_{\text {comp }}^{O}\right] \operatorname{diag}(1,1,1,1)$. Fig. 3 shows the resulting $\mathrm{MB}$ crash model for a full overlap frontal V2V collision. However, this simplified MB model can only deal with collision constellations that have a high overlap and the principal direction of the force is in line with the CG.

\subsubsection{Impulse and momentum (IM) crash model}

An inelastic vehicle collision can also be modeled with the law of conservation of momentum and the energy equations. The principle of impulse $\mathbf{I}$ :

$m\left(\mathbf{v}(t)^{\prime}-\mathbf{v}\left(t_{0}\right)\right)=\mathbf{I}$, 


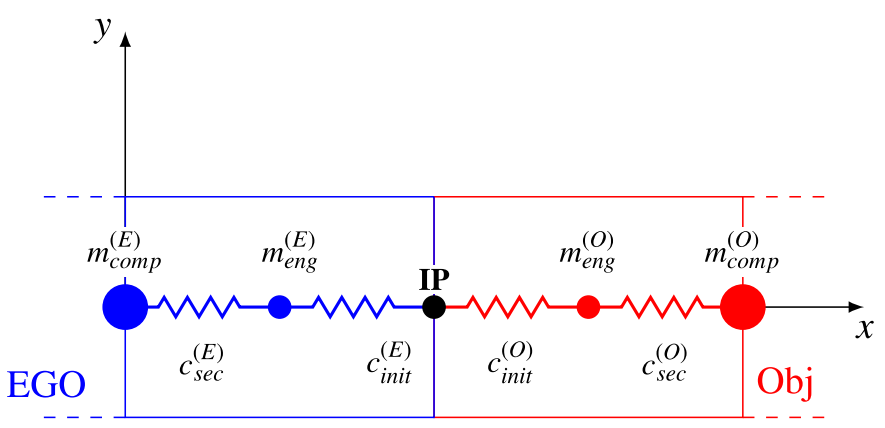

Fig. 3. MB crash model for full overlap frontal impact scenarios of two vehicles (top view) consisting of 4 springs and 4 masses connected at the impact point $\mathbf{I P}=\left[I P_{x}\right.$, $\left.I P_{y}\right]$.

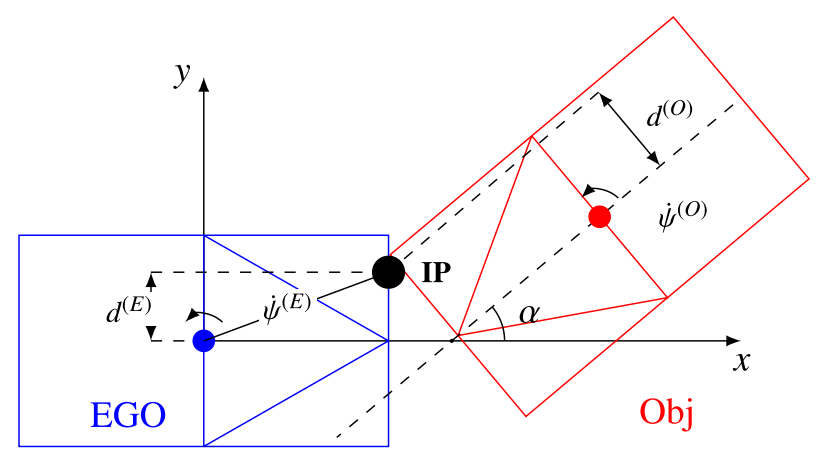

Fig. 4. Impulse and momentum crash model considering the impact point IP, collision angle $\alpha$ and rotational velocities $\dot{\psi}$.

and the change in the angular momentum $\mathbf{L}$ :

$\mathbf{L}(t)^{\prime}-\mathbf{L}\left(t_{0}\right)=\mathbf{J}\left(\boldsymbol{\omega}(t)^{\prime}-\boldsymbol{\omega}\left(t_{0}\right)\right)=\mathbf{r} \times \mathbf{I}=I P_{x} I_{y}-I P_{y} I_{x}$,

can be used to calculate the velocity $\mathbf{v}(t)^{\prime}$ and angular velocity $\boldsymbol{\omega}(t)^{\prime}=\left[0,0, \dot{\boldsymbol{\psi}}(t)^{\prime}\right]^{T}$ after collision, where $\mathbf{r}$ denotes the lever (distance to impact point IP), $t_{0}$ is the time of collision and $\mathbf{J}$ the inertia tensor. A collision can be divided into two phases: compression and restitution. In this work the coefficient of restitution $k$ is used to estimate the change of velocity $\Delta V^{E}$ of the ego vehicle. $k$ denotes the ratio of relative velocity of separation to the relative velocity before collision of two colliding objects (Brach and Brach, 2011). Fig. 4 shows a angular Front-Front collision of two vehicles. Based in Eq. (9) and (10), ego vehicle's change of velocity $\Delta V^{E}$ can be calculated for collisions with small collision angles $\left(I_{y}=0, v_{y, t_{0}}^{E}=v_{y, t}^{E^{\prime}}\right)$ in the $x y$-plane $\left(I P_{y}=d\right)$ :

$$
\begin{aligned}
& \Delta V_{x}^{E}=v_{x, t_{0}}^{E}-v_{x, t}^{E^{\prime}}, \\
& k=-\frac{v_{\mathbf{I P}, x, t}^{E^{\prime}}-v_{\mathbf{I P}, x, t}^{O^{\prime}}}{v_{\mathbf{I P}, x, t_{0}}^{E}-v_{\mathbf{I P}, x, t_{0}}^{O}}, \quad v_{\mathbf{I P}, x}=v_{x}-d \dot{\psi}, \\
& v_{x, t}^{E^{\prime}}=v_{x, t_{0}}^{E}-\frac{I_{x}^{E}}{m^{E}}, \\
& \dot{\psi}_{t}^{E^{\prime}}=\dot{\psi}_{t_{0}}^{E}+\frac{d^{E} I_{x}^{E}}{J_{z}^{E}}, \\
& I_{x}^{E}=(1+k) \cdot \frac{v_{x, t_{0}}^{E}-v_{x, t_{0}}^{O}-d^{E} \dot{\psi}_{t_{0}}^{E}+d^{O} \dot{\psi}_{t_{0}}^{O}}{\frac{1}{m^{E}}+\frac{1}{m^{O}}+\frac{\left(d^{E}\right)^{2}}{J_{z}^{E}}+\frac{\left(d^{O}\right)^{2}}{J_{z}^{O}}},
\end{aligned}
$$

where $I_{x}^{E}$ (Eq. (15)) is the momentum that acts on the ego vehicle along the $x$-axis and $v_{\mathbf{I P}, x}$ the vehicle's velocity at IP. $J_{z}^{E}$ and $J_{z}^{O}$, are the momentums around the vehicles $z$-axis respectively. The distances $d^{E}$ and $d^{O}$ are the $y$-distance between the IP and the CG of

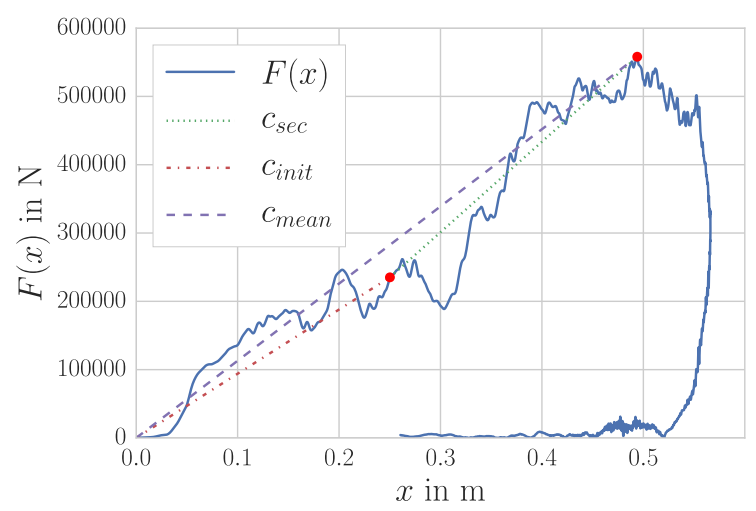

Fig. 5. Force over vehicle's front end crush intrusion.

each vehicle (in the case that there are different impact overlaps). The coefficient of restitution $k$ characterizes the in-elasticity of a collision. The coefficient of restitution $k$ denotes the ratio between kinetic energy before the collision and the energy resulting in permanent vehicle deformation as well as kinetic energy after the collision. Both models can not deal with bigger impact angles $\alpha$. For a detailed derivation of Eq. (11)-(15) and additional equation used for planar impact mechanics with higher impact angles $\left(I_{y} \neq 0\right)$ we refer to Raymond Brach and Matthew Brach (2007), Appel et al. (2002). Parameters from both colliding objects are needed to calculate a crash severity. It is investigated in the next section how these inputs can be derived from the objects shape.

\subsection{Crash model parameter correlation analysis}

The crash severity can be estimated using models and input parameters like stiffness, coefficient of restitution and the collision opponents mass. Since a forward-looking sensor can only deliver the vehicle dimensions, it is investigated how it correlates to crash models input parameters. Therefore the 40 vehicle frontal impact tests against load cell walls conducted by the NHTSA were studied NHTSA (2016). The force measurements over the vehicle's front end crush intrusion is used to calculate the vehicles front end stiffness (Fig. 5 shows the stiffness calculation using an impact test).

The data show that there is no significant correlation between the vehicle mean stiffness and its dimensions. The checker-board Fig. 7 shows the results of the correlation analysis. The color red indicates a significant correlation and the color blue a poor correlation between variables. It is conspicuous that there is no significant correlation between mass and mean stiffness (assuming a linear force on the deformation path). This result is also described in Nusholtz et al. (2005), Appel et al. (2002). It is difficult to define an exact vehicle frontal stiffness. There is no significant correlation between mass and mean stiffness for the vehicles, but a dependence between the initial stiffness and the vehicle mass. The initial stiffness increases with vehicle weight (Fig. 7). This result is conform with the outcome of Swanson's analysis (Swanson et al., 2003).

The data shows further good correlation between the vehicle mass and its width $w$, length $l$, and wheelbase $l_{b}$. Especially, the combination of the vehicles width and length (footprint $A$ ) as well as its wheelbase demonstrate good correlations between these variables and the vehicle mass. Fig. 6 shows the relationship between vehicle masses and dimensions. The blue points show the minimum vehicle weight and the red points the maximum vehicle mass over vehicle dimensions. The 40 vehicle frontal impact tests against load cell walls are further used to rate and compare the OLC estimation error using both MB crash models from Section 4.1.1. Compared to the model using only one mass and one global frontal stiffness the $O L C$ estimation error compared to the real crash pulse was in 


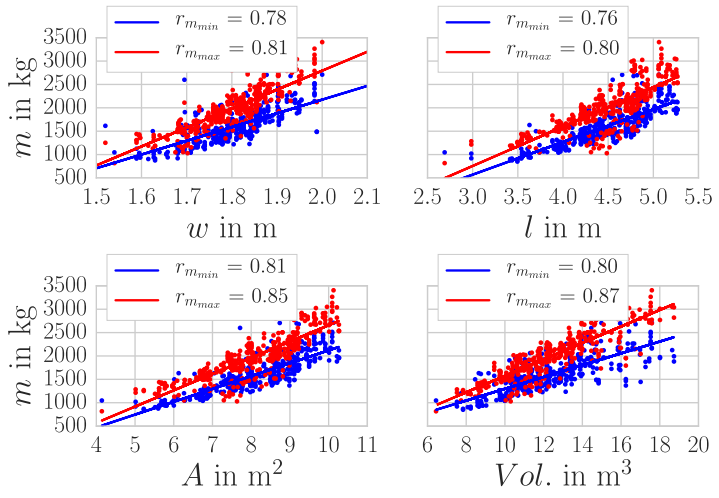

Fig. 6. Correlation between mass and vehicle dimensions.

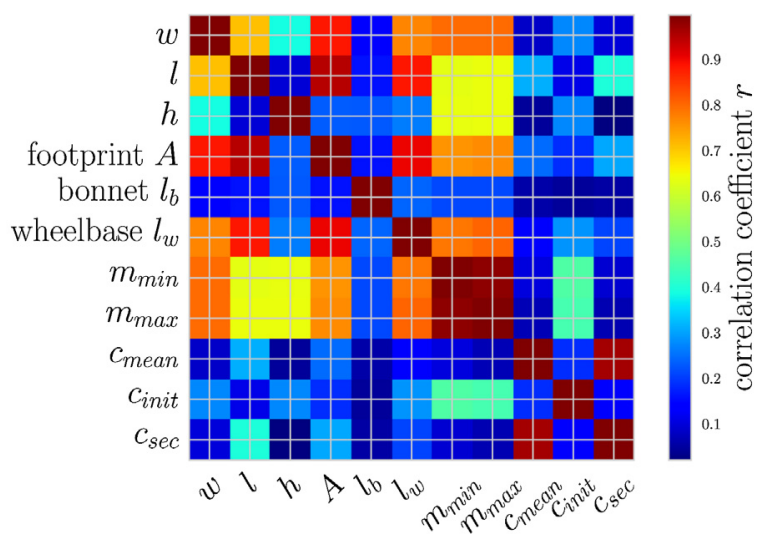

Fig. 7. Correlation between vehicle parameters.

average $47 \%$. The OLC estimation error could be improved by $32 \%$ on average with the model that assumes 2 linear stiffness and two masses for one vehicle front. In order to parameterize these models, the crash opponents stiffness and mass has to be estimated using exteroceptive sensor measurements. Burg presents in Burg (1982) the functional relationship between vehicles length, mass and its wheelbase in order to estimate the vehicles momentum of inertia $J_{z}$ around the $z$-axis. Since the wheel base $l_{w}^{O}$ is difficult to measure with exteroceptive sensors, the strong correlation found between the vehicle length and its wheelbase is used to adopt Burgs formula. In order to estimate $J_{z}$ a different constant factor is used to be able to substitute $l_{w}^{O}$ by $l^{O}$ :

$J_{z}^{O}=0.0863 m^{O}\left(l^{O}\right)^{2}$.

The coefficient of restitution $k$ is used to predict the change of velocity $\Delta V$ in vehicle collisions and can be estimated from crash test data (Monson and Germane, 1999). Previous studies show that the coefficient of restitution in V2V collisions shows strong dependence on vehicle collision speed and the overlap (Elbel, 2007; Kalthoff, 2009; Kalthoff and Becke, 2000; Appel et al., 2002). In this work, extracted Front-Rear V2V collisions with small impact angles and high overlaps form the German in-Depth Accident Study (GIDAS) database as well as V2V-FEM simulations with different vehicle types are utilized to calculate $k$. Fig. 8 shows the V2V crash constellations used in FEM simulations (Pam-Crash). Four different vehicle types were used in 3000 crash constellations. It is possible that the vehicles involved in collision get caught up, stick together or slip against each other. Figs. 9 and 10 show the resulting $k$ in dependence of the relative collision velocity $v_{t_{0}}^{\text {rel }}$, IP and $\alpha$ at time of collision for V2V collisions. Locally Weighted Scatterplot Smoothing (LOWESS) is used to create a smooth line through the plotted coefficients of restitution $k$ (Cleveland and Devlin, 1988).

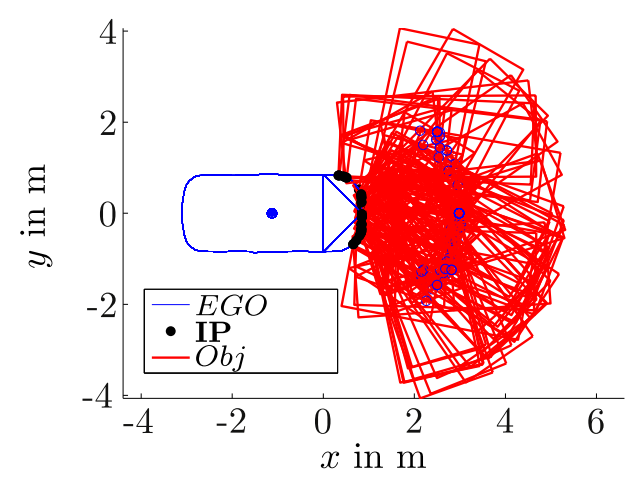

Fig. 8. Different vehicles and crash constellations are used in FEM simulations (PamCrash). Contours of colliding vehicles can be seen from top view in the $x$ - $y$-plane.

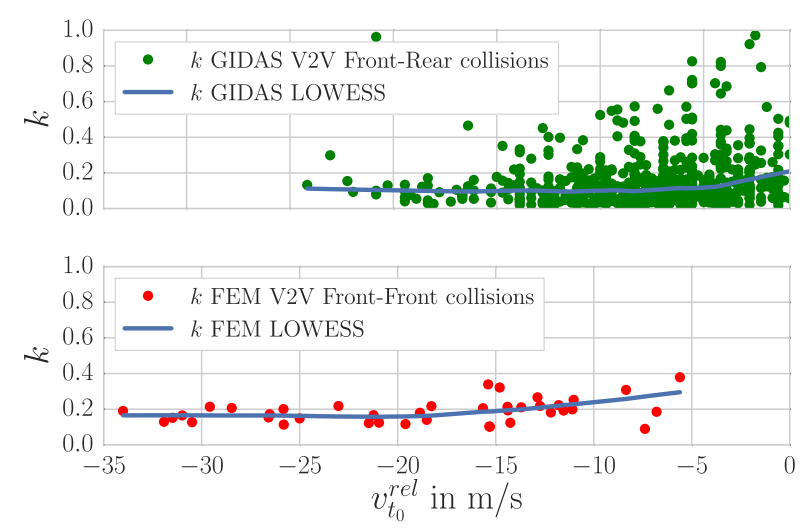

Fig. 9. In both figures calculated coefficients of restitution $k$ in dependence of the collision velocity $v_{t_{0}}^{\text {rel }}$ in V2V collisions can be seen. First figure shows reconstructed V2V front-rear collisions from the GIDAS database and the second figure shows V2V front-front collisions using data from FEM simulations. All used collisions have a full overlap $\left(I P_{y} \leq 65 \%, O L \geq 40 \%\right)$ and small collision angles $\left(\alpha \leq 15^{\circ}\right)$.
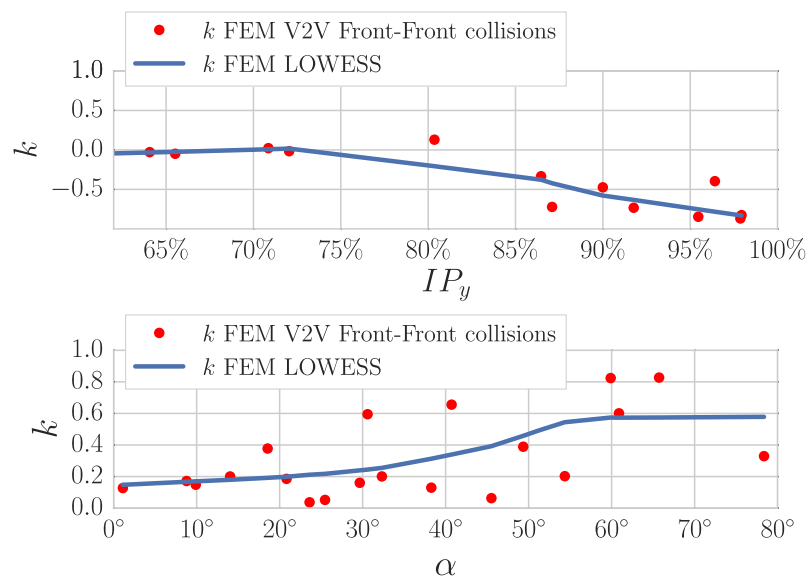

Fig. 10. First figure shows calculated coefficients of restitution $k$ in V2V collisions with small overlap ( $\left.I P_{y} \geq 65 \%, O L \leq 40 \%\right)$ using FEM simulations. Second figure plots the calculated $k$ in dependence of the collision angle $\alpha$ in collisions with high overlap $\left(I P_{y} \leq 65 \%, O L \geq 40 \%\right)$ and high velocities $\left(v_{t_{0}}^{\text {rel }} \geq 15 \mathrm{~m} / \mathrm{s}\right)$.

In Fig. 9 the LOWESS curve shows a similar quantitative relationship between $k$ and the relative impact velocity $v_{t_{0}}^{r e l}$ for a full frontal vehicle to barrier collisions found in literature (Appel et al., 2002). The restitution coefficient $k$ is negative for frontal collisions with small collision overlaps when the vehicles slip against each other. In Fig. $10, k$ is plotted over the distance of the impact point $I P_{y}$ expressed in \% to make the influence of collision overlap comparable for different car types: $I P_{y}=2\left(d^{E} / w^{E}\right) 100 \%$. When $v_{t_{0}}^{r e l}$ is 
sufficient high and $I P_{y}$ is getting bigger than $80 \%$, the two colliding vehicles glide past each other and not get caught up. Therefore, in these cases, the change in velocity $\Delta V$ is very low. However, there can be high intrusions and deformations. Fig. 10 also shows the influence of $\alpha$ on $k$ using the planar impact model found in (Raymond Brach and Matthew Brach, 2007). The calculated data shows that with increasing impact angles $\left(\alpha \geq 15^{\circ}\right)$ the scattering of $k$ is getting larger.

\section{Creation of crash severity data model (CSDM)}

The CSDM is used as a knowledge data base in the reasoning layer to rapidly derive triggering decisions in pre-crash situations. It allows to predict about the various final conditions of an initial pre-crash situation using the knowledge of possible crash severities and its probabilities. Therefore, the CSDM comprises findings deduced from accident data, crash severity and driver behavior models. On the basis of observational data the crash severity model parameters have to be estimated. In the following a methodology is presented to calculate the crash severity by means of actual ego sensor measurements, the estimated object state and a-prior knowledge (context information). The presented crash models (MB and IM) in Section 3 can be applied to calculate possible realizations of $C s(\triangle V$ and $O L C)$ for a specific crash constellation $\mathbf{S}$ at $t_{0}$ :

$C s_{\Delta V}=h_{I M}\left(\mathbf{S}_{I M}\right)=h_{I M}\left(k, v_{t_{0}}^{r e l}, m^{O}, \mathbf{I P}, O L, \alpha\right)$,

$C s_{O L C}=h_{M B}\left(\mathbf{S}_{M B}\right)=h_{M B}\left(v_{t_{0}}^{r e l}, \mathbf{M}^{O}, \mathbf{I P}, \mathbf{C}\right)$,

using predictions of $v_{t_{0}}^{r e l}, \mathbf{I P}, m^{O}, \alpha, O L, k$ or $\mathbf{C}$ at time of collision $t_{0}$. These predictions are not unique and depend on the driver behavior just before collision, measurement uncertainties and statistical relationships between the estimated variables and the measurements (e.g. the correlation between object mass $m^{O}$ and its estimated width $\hat{w}^{O}$ ).

Therefore, model parameters are derived from sensor measurements using their statistical relationship. The main objective is to reason about the possible crash in the presence of uncertainty based on the estimated object state using current and past sensor measurements. Therefore the crash severity is considered as a continuous random variable $C s$ conditioning on $\hat{\mathbf{x}}_{n \mid n}$, the estimated state vector at time $n$ before an unavoidable collision. The estimated object state $\hat{\mathbf{x}}_{n \mid n}$ consists of the object's width estimation $\hat{w}_{n \mid n}^{O}$, the relative distance to the object $\mathrm{r}=\left[\hat{r}_{x}, \hat{r}_{y}\right]$ and the relative velocity $\mathrm{v}^{\text {rel }}=\left[\hat{v}_{x}^{\text {rel }}, \hat{v}_{y}^{r e l}\right]$ based on the actual (at time step $n$ ) and previous measurements. The expected crash severity measure depends on the crash constellation $\mathbf{S}$ at $t_{0}$ and can be calculated as follows:

$\mu_{\mathbf{S} \mid \hat{\mathbf{x}}_{n \mid n}}=\int h(\mathbf{S}) f\left(\mathbf{S} \mid \hat{\mathbf{x}}_{n \mid n}\right) d \mathbf{S}$

$$
\begin{aligned}
f\left(\mathbf{S}_{I M} \mid \hat{\mathbf{x}}_{n \mid n}\right)= & f\left(k, v_{t_{0}}^{r e l}, m^{O}, \mathbf{I P}, O L, \alpha \mid \hat{\mathbf{x}}_{n \mid n}\right),=f\left(k, v_{t_{0}}^{r e l}, m^{O}, \mathbf{I P}, O L,\right. \\
& \left.\alpha \mid \mathbf{r}_{n \mid n}, v_{n \mid n}^{r e l}, \hat{w}_{n \mid n}^{O}\right),=f\left(m^{O} \mid \hat{w}_{n \mid n}^{O}\right) \cdot f\left(k, v_{t_{0}}^{r e l}, \mathbf{I P}, O L,\right. \\
& \left.\alpha \mid \mathbf{r}_{n \mid n}, v_{n \mid n}^{r e l}, \hat{w}_{n \mid n}^{O}\right),
\end{aligned}
$$

with:

$$
\begin{aligned}
& f\left(k, v_{t_{0}}^{r e l}, \mathbf{I P}, O L, \alpha \mid \mathbf{r}_{n \mid n}, \mathbf{v}_{n \mid n}^{r e l}, \hat{w}_{n \mid n}^{O}\right)=\int f\left(k, v_{t_{0}}^{r e l}, \mathbf{I P}, O L, \alpha, \tau^{E},\right. \\
& \left.\tau^{O} \hat{\mathbf{x}}_{n \mid n}\right) d \tau^{E} d \tau^{O},=\int f\left(k, v_{t_{0}}^{r e l}, \mathbf{I P}, O L, \alpha \mid \tau^{E}, \tau^{O}\right) \\
& \cdot f\left(\tau^{E}, \tau_{\mid \hat{\mathbf{x}}_{n \mid n}}\right) d \tau^{E} d \tau^{O} .
\end{aligned}
$$

In order to determine $\mu_{\mathbf{S} \mid \hat{\mathbf{x}}_{n \mid n}}$ for a measured pre-crash scenario $\hat{\mathbf{x}}_{n \mid n}$, the probability density functions (PDF) $f\left(\tau^{E}, \tau^{O} \mid \hat{\mathbf{x}}_{n \mid n}\right)$, $f\left(k, v_{t_{0}}^{\text {rel }}, \mathbf{I P}, O L, \alpha \mid \tau^{E}, \tau^{O}\right)$ and $f\left(m^{O} \mid \hat{w}_{n \mid n}^{O}\right)$ have to be determined. The object's width $w^{O}$ is selected, since it is measurable with a Lidar sensor and has a good correlation with the objects mass in the case the potentially-colliding obstacle is a vehicle (see Figs. 6 and 7).

The decision process can be modeled as a transfer function $g\left(\mu_{\mathbf{S} \mid \hat{\mathbf{x}}_{n \mid n}}\right)$ that maps the expected crash severity $\mu_{\mathbf{S} \mid \hat{\mathbf{x}}_{n \mid n}}$ based on the current sensor measurements to a safety actuator triggering strategy. E.g. the TS of an airbag system is modeled with three states: No Fire $N F$, Can Fire $C F$ (deployment accepted, not required) and Must Fire $M F$. The decision function can be expressed as follows:

$$
\begin{aligned}
T S= & g\left(\mu_{\mathbf{S} \mid \hat{\mathbf{x}}_{n \mid n}}\right), \\
& \text { with : } \\
& \hat{\mathbf{x}}_{n \mid n}=\left[\mathrm{r}_{n \mid n}, \mathrm{v}_{n \mid n}^{r e l}, \hat{w}_{n \mid n}^{O}\right]=\left[\mathrm{r}_{n \mid n},\left\|\mathbf{v}_{n \mid n}^{r e l}\right\|, \hat{\psi}_{n \mid n}^{O}, \hat{w}_{n \mid n}^{O}\right], \\
& \text { and : } \\
& T S \in\{N F, C F, M F\} .
\end{aligned}
$$

The decision function $g\left(\mu_{\mathbf{S} \mid \hat{\mathbf{x}}_{n \mid n}}\right)$ is based on the knowledge stored in the CSDM. In the following, the PDFs $f\left(m^{O} \mid \hat{w}_{n \mid n}^{O}\right)$, $f\left(k, v_{t_{0}}^{\text {rel }}, \mathbf{I P}, O L, \alpha \mid \tau^{E}, \tau^{O}\right)$ (crash model and accident data) and $f\left(\tau^{E}, \tau^{O} \mid \hat{\mathbf{x}}_{n \mid n}\right)$ (driver model) are numerical determined. $\mu_{\mathbf{S} \mid \hat{\mathbf{x}}_{n \mid n}}$ is calculated for a defined set of associated $\hat{\mathbf{x}}_{n \mid n}$ using sampling from the PDFs.

\subsection{Accident data used in the CSDM}

The CSDM combines accident databases, the crash severity models, driver behavior models and sensor/tracking models to calculate possible $C s$ and the associated TS for different pre-crash scenarios. In dependence of the estimated state $\hat{\mathbf{x}}_{n \mid n}$, the CSDM is created using the statistical properties found in the data and models. Of particular interest is the unknown $\operatorname{PDF} f\left(C s \mid \hat{\mathbf{x}}_{n \mid n}\right)$ in dependence of the estimated state $\hat{\mathbf{x}}_{n \mid n}$ that allows to calculate the expected crash severity and find probabilities $f$ associated with possible crash scenarios $\left(\left(\tau_{i}^{(E)}, \tau_{j}^{(O)}\right)\right)$ and $C s_{i j}$. A PDF can be directly estimated with numerical methods, for instance the kernel or the nearest neighbor method, from observed data (Silverman, 1986). At this point it is omitted and referred to Jacobs (2009) to find the PDFs analytical form. In this work sample data is used to estimate $\tilde{f}\left(C s \mid \hat{\mathbf{x}}_{n \mid n}\right)$ for each input $\hat{\mathbf{x}}_{n \mid n}$. The sampling is based on statistical data extracted from different crash databases, driver behavior studies, sensor and tracking models.

\subsubsection{Vehicle (Crash) data acquisition}

The underlying data consists of data extracted from the NHTSA crash test data base (full frontal vehicle collisions against a rigid load cell wall), GIDAS database (frontal straight head on V2V collisions with small impact angles) and FEM simulations (using the software package Pam-Crash). In order to predict the Cs the restitution coefficient $k$ is calculated from the crash data. $\tilde{f}\left(k \mid v_{t_{0}}^{\text {rel }}, d^{E}\right) \approx$ $f\left(k \mid v_{t_{0}}^{r e l}, m^{O}, \mathbf{I P}, O L, \alpha\right)$ can be estimated based on the extracted crash data and the kernel density method for small collision angles. For simplification, higher impact angles ( $\alpha \geq 15^{\circ}$ see Fig. 7 ), are not considered since the prediction would get very uncertain. Fig. 11 shows the resulting PDF. Information about dimensions and masses have been taken from vehicle blue prints, the GIDAS data and the NHTSA crash test data (see Fig. 6) to estimate $\tilde{f}\left(m^{0} \mid w\right.$ ), since the width $w$ shows good correlation with the mass of an vehicle and is always measurable in rear- or front collisions. Fig. 12 shows the estimated $\operatorname{PDF} \tilde{f}\left(m^{O} \mid w\right)$ using kernel density method with a Gaussian kernel. 


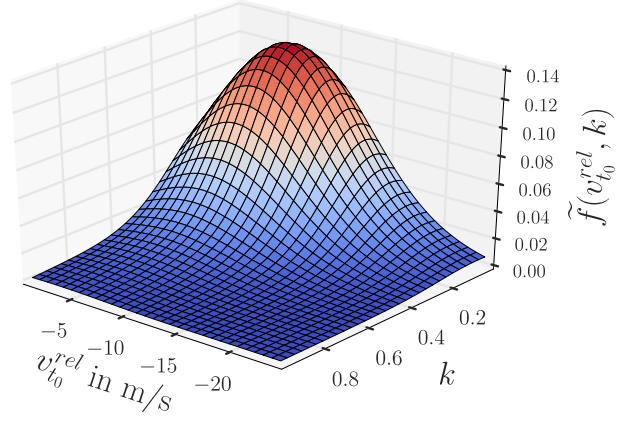

Fig. 11. Estimated PDF on the vehicle database using a Gaussian kernel.

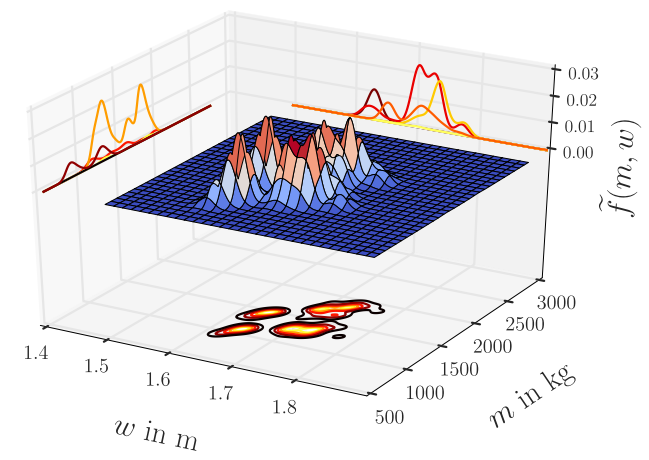

Fig. 12. Estimated PDF on the vehicle database (weighted mass width distribution for German roads) using a Gaussian kernel and the related contour plot.

\subsubsection{Driver behavior model for collision velocity prediction}

It is necessary to consider the driver behavior in the crash severity prediction. Possible braking and/or steering maneuvers just before a collision can influence the crash model input parameters. A braking maneuver, for example, does reduce the collision speed at collision and thus the crash severity. Under normal cruise and small time intervals it can be considered that the driver does not apply big acceleration changes. If a collision is imminent and the driver is alerted, it is more likely that the driver does suddenly decelerate and/or steer (McGehee and Carsten, 2010; Weber, 2015). However, the literature shows also that in the majority of accidents, the driver does not react at all or does not use the full vehicle's maneuvering capability in order to avoid or to mitigate the imminent collision (Langwieder, 2013; Eckert et al., 2011; Wiacek, 1999). For a good driver behavior prediction in pre-crash scenarios the driver attention has to be monitored very closely and constantly. Since it was not possible for this investigation to conduct a study to analyze the driver behavior in critical pre-crash scenarios, analysis results found in literature serve as data basis to generate a maneuvering PDF. The driver alertness is modeled to be evenly distributed between being inattentive and alert. So in $50 \%$ the cases the driver is attentive and will react in the same way like described in (Weber, 2015; Langwieder, 2013; Wiacek, 1999). Mrs. Weber analyzed the driver behavior in four scenarios with different TTCs ( $1.6 \mathrm{~s}, 1.4 \mathrm{~s}, 1.2$ $\mathrm{s}$ and $1.0 \mathrm{~s}$ ). This data is not sufficient for the purpose of our work because the driver behavior for TTCs smaller then $1 \mathrm{~s}$ is relevant. So the principal tendency found in the findings is used to approximate the driver behavior in scenarios with lower TTCs. In addition, driving dynamic limits were chosen: the circle of forces (physical traction limits between tier and road surface) and the fact that the fewest drivers use the entire possible applicable accelerations in the $x-y$-plane to avoid collisions (Wegscheider and Prokop, 2005). In order to estimate $\tilde{f}\left(v_{t_{0}}^{\text {rel }} \mid \hat{\mathbf{x}}_{n \mid n}\right)$ the driver behavior is modeled with $\operatorname{PDF} f\left(\tau^{E}, \tau^{O} \mid \hat{\mathbf{x}}_{n \mid n}\right)$. The KDE is used to estimate $\tilde{f}\left(a_{x}, a_{y} \mid T T C\right)$. Each possible trajectory $\tau^{E}$ and $\tau^{O}$ can be modeled in dependence of the
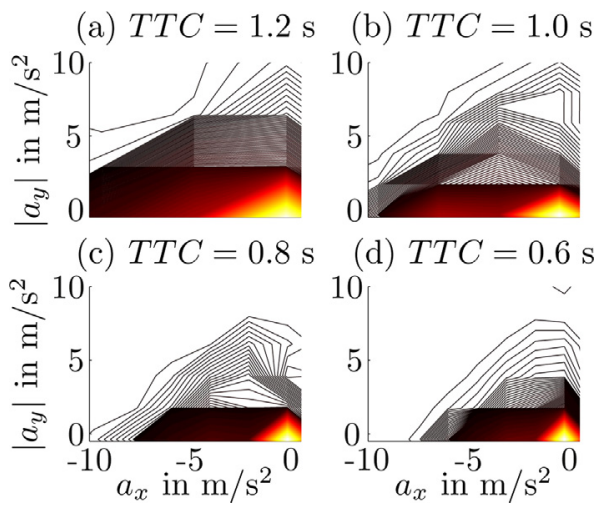

Fig. 13. Contour plot of the chosen accelerations in the $x-y$-plane in pre-crash scenarios based on the estimated PDFs $\tilde{f}\left(a_{x}, a_{y} \mid T T C\right)$ for four different TTCs using data from literature Weber (2015), Langwieder (2013), Wiacek (1999).

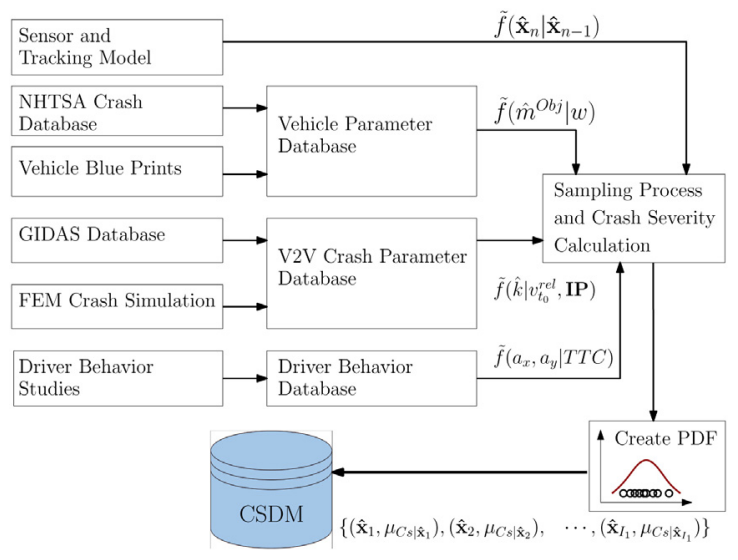

Fig. 14. Sampling methodology in order to create the CSDM.

TTC (driver model). Fig. 13 shows the contour plot (top view of isolines) of the generated PDF chosen accelerations $a_{x}$ and $a_{y}$ in the $x-y$-plane for four different TTCS.

\subsection{Sampling methodology and resulting model}

In the beginning, possible estimated state vectors $\hat{\mathbf{x}}_{n \mid n}$ are generated. Uniformly distributed pseudo-random numbers (every possible state occurs with the same probability) of combinations of $\mathrm{r}$ distance and relative velocity $\mathrm{v}^{\text {rel }}$, that satisfies the collision unavoidable condition:

$\operatorname{TTC}_{u}=\frac{\|\mathrm{r}\|}{\left\|\mathrm{v}^{r e l}\right\|} \leq 1 \mathrm{~s}$,

are generated. The width $\hat{w}_{n}^{O}$ is also uniformly distributed in the interval $\hat{w}_{n \mid n}^{O} \in[1.5 \mathrm{~m}, 2.5 \mathrm{~m}]$. For each of the resulting state vectors (pre-crash situation) $\hat{\mathbf{x}}_{n \mid n, i_{1}}$ with $i_{1} \in\left[1, \ldots, I_{1}\right]$ the expected crash severity $\mu_{C s \mid \hat{\mathbf{x}}_{n \mid n}}$ is calculated. In Fig. 14 the different databases and models used can be seen.

Based on the above estimated PDFs $\tilde{f}\left(k \mid v_{t_{0}}^{r e l}, d^{E}\right), \tilde{f}\left(m^{O}, w^{O}\right)$ and $\tilde{f}\left(a_{x}, a_{y} \mid T T C\right)$ as well as the Gaussian PDFs of the estimated object state vectors (predicted covariance resulting from sensor and process noise), the expected crash severities for each state vector $i_{1}$ can be calculated. The calculation of the crash severities can be done by sampling the crash model input parameters. The data samples are generated using the input parameters PDFs and a random selection process. To determine the PDF $\tilde{f}\left(C s \mid \hat{\mathbf{x}}_{n \mid n}\right)$ and the expected crash severity $\mu_{C s \mid \hat{\mathbf{x}}_{n \mid n}}$ for one scenario $i_{1}$ a finite number $I_{2}$ of possible crash severities $C s_{1}, C s_{2}, \ldots, C s_{I_{2}}$ are calculated. Pseudo-random 


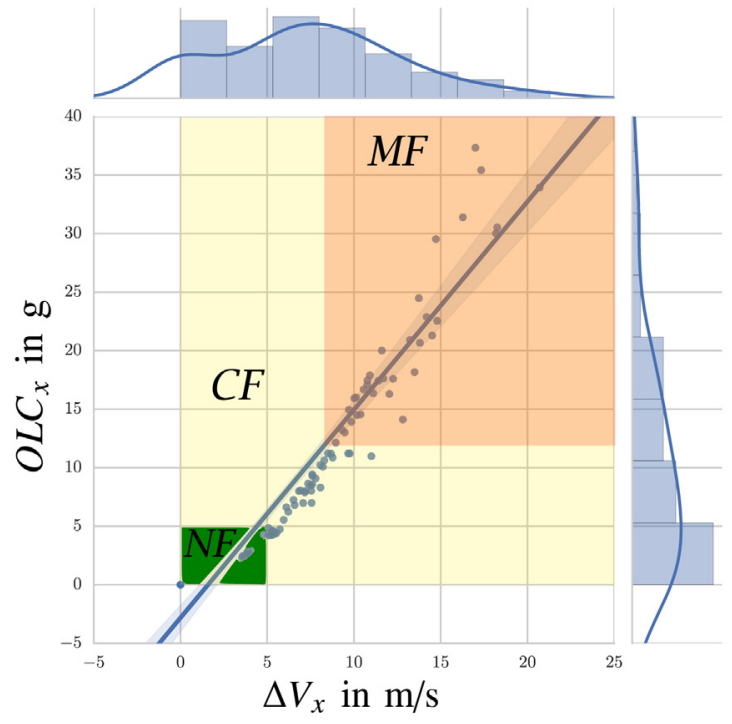

Fig. 15. Relationship between $O L C_{x}$ and $\Delta V_{x}$ based on V2V FEM crash simulations. Three triggering strategy TS classes ( $N F$ green area, $C F$ yellow area and $N F$ red area) based on occupant injury thresholds and vehicle damage pattern. (For interpretation of the references to color in this figure legend, the reader is referred to the web version of the article.)

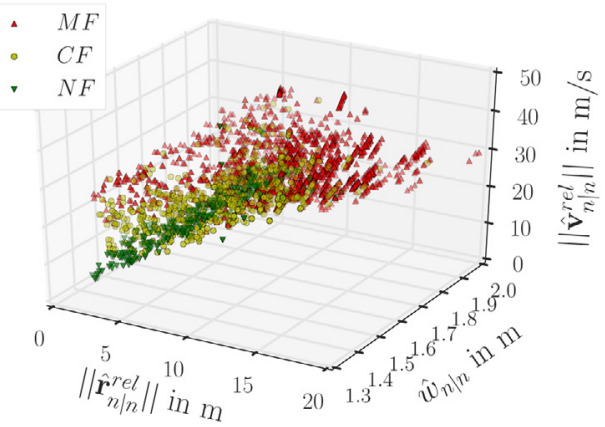

Fig. 16. Generated feature space defines a mapping of an estimated state $\hat{\mathbf{x}}_{n \mid n}$ to a triggering strategy $T S$.

numbers are generated according to the $i_{1}$ th estimated state vector and the PDFs in order to calculate possible crash severities. Random numbers can be generated using the rejection sampling or Markov Chain Monte Carlo (MCMC) methods. Possible Cs realizations are calculated with the impulse and momentum crash model Eq. (11) in Section 3, where the input parameters are sampled according their PDFs. Depending on the choice of the quantity of random numbers used, $I_{2}$ possible crash severities are calculated (here for each state vector $I_{2}=2^{31}$ crash severities are calculated for each estimated state vector $\hat{\mathbf{x}}_{n \mid n}$ ). According to the calculated crash severities the PDF can be estimated. That means all possibilities of the crash outcome are calculated under the condition that the state vector $\hat{\mathbf{x}}_{n \mid n}$ has been estimated. Finally the expectation of $C s$ can be assigned to an triggering strategy TS. This represents a simple classification task. For example, the functional relationship between $O L C$ and $\Delta V$ (seen in Fig. 15) can be used to determine the associated triggering strategy class. A combined OLC and $\Delta V$ threshold for airbag activation based on V2V FEM crash simulations is used to label each state vector $\hat{\mathbf{x}}_{n \mid n, m}$ and crash outcome with the corresponding safety actuator triggering strategy $T S=\{N F, C F, M F\}$. Consequently, for each of state vector $i_{1}$ a respective TS can be assigned. This gives rise to a five-dimensional space (feature space), which maps to a state vector $i_{1}$ a corresponding TS. The resulting feature space is shown in Fig. 16. Finally, hyperplanes can be used to model the transfer function $g\left(\mathbf{x}_{n \mid n}\right)$. These hyperplanes separate the feature space in sub spaces that are associated to the different TS classes. The hyperplanes can be practically applied by expertise and experiments or with machine learning techniques. For example, a Support Vector Machine (SVM) can be used in this case to fit hyperplanes in this feature space. The resulting feature space reflects the complexity of a triggering decision. In this form the TS is deterministic and understandable, hyperplanes can interpreted and modified by humans. Furthermore, Cs calculation is based on a physical model, so there is a near-natural relationship between model and reality. Only defined and reproducible conditions can occur.

\section{Results and discussion}

First the training results of the SVM on the CSDM are presented. Then a methodology is introduced to simulate and evaluate the above-presented context-aware system using real accident data. The simulation is for re-enactment of real V2V collisions from its pre-crash phase up to time of collision. The triggering decisions are evaluated against the real (reference) collision outcome. The simulation includes sensor fusion, tracking and crash prediction based on real accident data and different sensor models.

\subsection{Training results}

A SVM with Gaussian Radial Basis Function (RBF) kernel shows the best performance with a score of $95 \%$ to identify the sub spaces (scikit-learn (Pedregosa et al., 2011) a machine learning framework in Python is used to train and validate the SVM). To measure the classification accuracy, the recall and precision is evaluated and the best set of parameters is chosen. Recall is defined in the sense of a binary triggering classification. Positive and negative prediction compared to true condition: False Positives (FP) and False Negatives (FN). Precision and recall are inversely related and can be calculated as follows:

recall $=\frac{\mathrm{TP}}{\mathrm{TP}+\mathrm{FN}}$,

precision $=\frac{\mathrm{TP}}{\mathrm{TP}+\mathrm{FP}}$.

For the best classification performance a good trade-off between precision and recall needs to be achieved. Therefore, an unknown estimated state vector $i_{1}$ can be mapped to a TS accordingly to the resulting sub spaces. The $\mathrm{F}_{1}$ score $(\beta=1)$ is a weighted average of the precision and recall:

$\mathrm{F}_{1}=\frac{2 \cdot \text { precision } \cdot \text { recall }}{\text { precision }+ \text { recall }}$,

whereby $F_{1} \in[0,1]$ and indicates the best classification performance at the value 1 . Since the problem is a multi-class classification, precision, recall and $\mathrm{F}_{1}$ can be applied to each class independently. For evaluation the performance of the selected hyper-parameters and trained model is measured on a dedicated evaluation set that was not used during the model selection step. Evaluated parameters are: penalty parameter $C$ of the error term, degree of the polynomial Kernel function and RBF kernel coefficient $\gamma \cdot \gamma$ is used to define the influence of a single training example. The classifier is optimized by $\mathrm{F}_{1}$, which is done using grid search on the data set that comprises only $25 \%$ of the available data generated. Compared to other SVM kernels, the RBF kernel with C= $1 \cdot 10^{3}$ and $\gamma=1$ shows best performance with a $\mathrm{F}_{1}$-score of 0.96 to identify the sub spaces. Finally, when a collision is regarded as unavoidable, an unknown state vector $\hat{\mathbf{x}}$ can be mapped to a TS accordingly to the resulting sub spaces. In Fig. 17 the Receiver Operating Characteristic (ROC) for three TS classes: NF, CF and MF is shown. The ROC illustrates the performance of a classifier. Points 


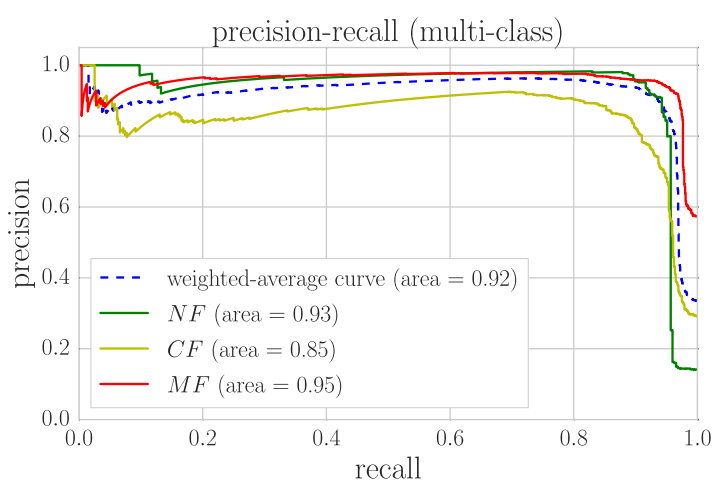

Fig. 17. The ROC (precision-recall curve) for the three TS classes.

above the diagonal ( $45^{\circ}$ orthogonal line) represent good classification results and the Area Under the Curve (AUC $\in\{0,1\}$ ) is a quality measure, whereby AUC $=1$ would be the best result. The area under the weighted-average curve is AUC $=0.92$ for the trained SVM.

\subsection{Reference data acquisition for simulation}

The GIDAS accident data base, that includes traffic accidents involving personal injury, is selected as input for the simulation. 200 real V2V crash scenarios with their extent of damage are extracted. The GIDAS data includes the course of accident and the exact description of the vehicles involved. Further, the accident data basis contains all relevant crash data at the time of impact (e.g. $v_{t_{0}}^{\text {rel }}$, $\alpha$, etc.) and the resulting $\Delta V$ that has been reconstructed after collision. But the GIDAS accident data base does not include information about the pre-crash phase. Hence, the exact vehicle maneuvers just before collision is unknown. Possible pre-crash ego and object trajectories $\tau$ are generated that lead to the GIDAS' crash constellations $\mathbf{S}_{G}$.

The initial ego and object state vectors $\mathbf{x}_{n}$ before collision are reconstructed using a single-track motion model that is calculated inverse. The final vehicle states $\mathbf{x}_{\mathbf{S}_{G}}\left(t_{0}\right)$ are given and the initial states $\mathbf{x}_{\mathbf{s}_{G}}\left(t_{n}\right)$ at time $t_{n}=t_{0}-\Delta t$ before collision have to be found. To calculate possible trajectories this end value problem is transformed into an initial value problem using substitution and the theoretical symmetry of physical laws under a time reversal transformation $T: \Delta t \rightarrow-\Delta t$ (Christiaans, 2004). The formulated approach is chosen to solve the differential equations of the singletrack motion model $f_{S T}$ (Schofield, 2008) and to keep the physical process despite the change of time direction. On the basis of the known GIDAS scenarios $\mathbf{S}_{G}$ at time $t_{0}$, random trajectories and state vectors can be generated by sampling the vehicles' pre-crash accelerations $\mathbf{a}(t)$ :

$\mathbf{S}_{G}=f_{S T}\left(\mathbf{x}\left(t_{n}\right), \mathbf{a}(t)\right) \rightarrow \mathbf{x}_{\mathbf{S}_{G}}\left(t_{n}\right)=-f_{S T}\left(\mathbf{S}_{G}, \mathbf{a}(t)\right)$.

The acceleration $\mathbf{a}(t)$ is simple random sampled from a set of possible accelerations $\mathbf{a}(t) \in\left[\mathbf{a}_{\min }, \mathbf{a}_{\max }\right]$. In order to maintain driving stability, $\mathbf{a}_{\min }$ and $\mathbf{a}_{\max }$ depend on the vehicle velocity $\mathbf{v}$ and on the coefficient of friction between the tires and the road (Schimmelpfennig and Hebing, 1982). For verification the methodology was applied to reconstruct precisely measured trajectories from driving tests. The error is smaller than $10^{-6} \mathrm{~m}$ in the reconstructed constellation $\mathbf{S}_{G}$ for trajectories with the assumption of constant acceleration. The smallest errors occur at low speeds and small steering angles. In this work three possible trajectories were generated for each of the 200 exported GIDAS V2V collisions resulting in 600 reference data sets for simulation with $\Delta t=2 \mathrm{~s}$.
Table 1

Sensor specifications used in simulation.

\begin{tabular}{llllllll}
\hline Sensor & $\sigma_{r_{x}}$ & $\sigma_{r_{y}}$ & $\sigma_{v_{x}}$ & $\sigma_{v_{y}}$ & AOV & $r_{\min }$ & $r_{\max }$ \\
\hline Lidar 1 & $0.1 \mathrm{~m}$ & $0.1 \mathrm{~m}$ & - & - & $110^{\circ}$ & $0.3 \mathrm{~m}$ & $100 \mathrm{~m}$ \\
Lidar 2 & $0.03 \mathrm{~m}$ & $0.03 \mathrm{~m}$ & - & - & $360^{\circ}$ & $0.3 \mathrm{~m}$ & $100 \mathrm{~m}$ \\
Radar & $0.06 \mathrm{~m}$ & $0.06 \mathrm{~m}$ & $0.02 \mathrm{~m} / \mathrm{s}$ & $0.02 \mathrm{~m} / \mathrm{s}$ & $150^{\circ}$ & $1 \mathrm{~m}$ & $50 \mathrm{~m}$ \\
\hline
\end{tabular}

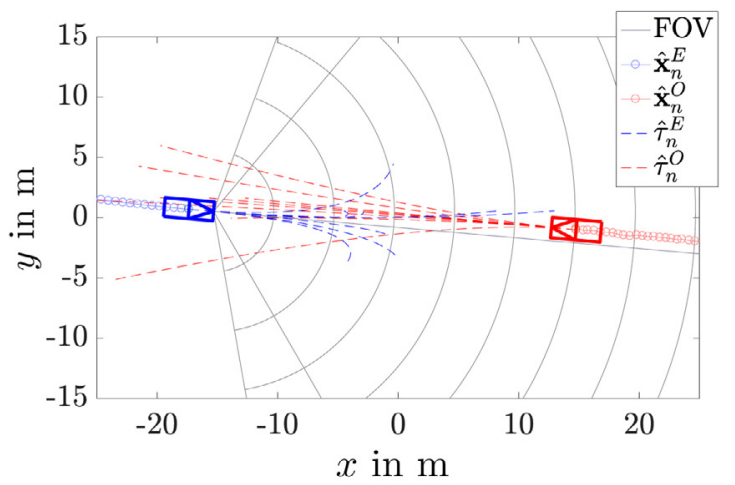

Fig. 18. Simulated GIDAS accident scenario using a SVF of Lidar and Radar sensor. The sensors Field of View (FOV) is shown and predicted trajectories $\hat{\tau}$ are plotted as dashed lines.

Table 2

Mean of relative errors.

\begin{tabular}{lllll}
\hline Meas. & Lidar 1 & Lidar 2 & SVF 1 & SVF 2 \\
\hline$r_{x}$ & $17.81 \%$ & $13.07 \%$ & $5.06 \%$ & $\mathbf{3 . 5 4} \%$ \\
$r_{y}$ & $29.38 \%$ & $21.62 \%$ & $12.42 \%$ & $\mathbf{1 0 . 0 4} \%$ \\
$v_{x}$ & $3.45 \%$ & $2.84 \%$ & $0.52 \%$ & $\mathbf{0 . 4 9} \%$ \\
$v_{y}$ & $4.66 \%$ & $4.91 \%$ & $2.19 \%$ & $\mathbf{2 . 0 2} \%$ \\
$w^{O}$ & $8.26 \%$ & $\mathbf{5 . 3 1} \%$ & $8.26 \%$ & $\mathbf{5 . 3 1} \%$ \\
$T \hat{T} C_{500}$ & $54.31 \%$ & $38.46 \%$ & $7.75 \%$ & $\mathbf{6 . 8 4} \%$ \\
\hline
\end{tabular}

Bold values indicate the smallest relative errors.

\subsection{Simulation}

The context-aware system consisting of sensor fusion, tracking and the crash prediction algorithm is implemented in Matlab for numerical simulation. Two sensor models for Lidar and Radar measurements are used for simulation. Each sensor is specified by a horizontal Angle of View (AOV), minimum range $r_{\min }$ and maximum range $r_{\max }$, a sampling interval and measurement noise. To obtain the sensor measurements, white Gaussian noise with the variance $\sigma$ is added if an obstacle is in the sensors field of view. To compare different sensor specifications and their influence on the prediction and triggering strategy outcome, two different Lidar sensors (Continental, 2015; Velodyne, 2015) and one Radar sensor (Ibeo Automotive Systems GmbH, 2015) are simulated (see Table 1). For simplification the sampling interval is chosen for each sensor to be the same $T_{S}=0.04 \mathrm{~s}$. The overall simulation duration of each scenario is $t_{\text {sim }}=2 \mathrm{~s}$. In Fig. 18 the top view of a simulated GIDAS scenario shortly before collision is shown.

\subsection{Discussion}

The context-aware system using the SVM trained on the CSDM is evaluated based on the simulated GIDAS V2V collisions. The system input condition is the detection of an unavoidable frontal V2V collision. Table 2 shows the mean of relative predicted states errors at $T T C=0.5 \mathrm{~s}$ in all simulated scenarios. It is evident that the SVF shows best results in relative distance, velocity and collision estimation. The possible triggering timing plays a major role for an airbag inflation. It shows that the collision was detected as unavoidable, with a SVF 2 using Lidar 2 and the Radar sensor, at a meantime 
Table 3

Error analysis of TS decision result on GIDAS V2V simulation.

\begin{tabular}{lllllll}
\hline True value (GIDAS) & Prediction & Error & Lidar 1 & Lidar 2 & SVF 1 & SVF 2 \\
\hline $\mathrm{MF}$ & $\mathrm{NF}$ & $\mathrm{FN}$ & 1 & 0 & 1 & 0 \\
$\mathrm{NF}$ & $\mathrm{MF}$ & $\mathrm{FP}$ & 2 & 1 & 1 & 0 \\
$\mathrm{CF}$ & $\mathrm{NF}$ & $\mathrm{FN}_{C}$ & 55 & 28 & 33 & 18 \\
$\mathrm{CF}$ & $\mathrm{MF}$ & $\mathrm{FP}_{C}$ & 55 & 29 & 18 & 6 \\
$\mathrm{NF}$ & $\mathrm{CF}$ & $\mathrm{FP}_{N}$ & 6 & 13 & 9 & 10 \\
$\mathrm{MF}$ & $\mathrm{CF}$ & $\mathrm{FN}_{M}$ & 1 & 3 & 25 & 10 \\
& & $\mathrm{Sum}$ & 120 & 74 & 87 & $\mathbf{4 4}$ \\
\hline
\end{tabular}

of $\overline{T T C} C_{u}=0.36 \mathrm{~s}$ before collision. The possible deployment time $t_{F}$ before collision depends on the estimated $T T C_{u}$, system latency $t_{l}$ and computing time $t_{\text {algo }}$ that the algorithm needs to interpret the situation. $t_{F}$ can be estimated as follows:

$t_{F}=T T C_{u}-t_{\text {algo }}-t_{l}$.

The simulation results show an average $\bar{t}_{F}=0.29 \mathrm{~s}$ using the SVF 2 with constant $T_{S}=0.04 \mathrm{~s}, t_{\text {algo }}=0.02 \mathrm{~s}$ and $t_{l}=5 \mathrm{~ms}$. The resulting $t_{F}$ is not normal distributed. The deviation of $t_{F}$ can be expressed using the 25 th percentile $t_{F}^{25 \text { th }}=0.21 \mathrm{~s}$ and 75 th percentile $t_{F}^{75 \text { th }}=0.38 \mathrm{~s}$ (values below which $25 \%$ or $75 \%$ of the simulated observations are found).

The trained SVM classifies a TS according to the predicted object and ego state at the time if a V2V collision $\left(T T C_{u}\right)$ is considered as unavoidable. Table 3 shows the classification results of all GIDAS scenarios simulations using different sensor sets and sub feature spaces created by a SVM with Gaussian RBF kernel. The SVF shows best results with no FP, no FN and 44 minor classification errors for all 600 simulated scenarios (error rate $=7.3 \%$ ). To achieve this results, randomly $10 \%$ of the GIDAS scenarios were added to the SVM training set. It is evident, that in selected pre-crash scenarios the triggering decision can be made even in the presence of uncertainty.

\section{Conclusion}

The severity of a vehicle collision can be significantly reduced by triggering safety systems prior to the collision. As result the risk of injury of occupants or VRUs can be decreased. Therefore potential collisions have to be identified by analyzing exteroceptive sensor measurements. This work deals with the question how triggering decisions can be made shortly before an unavoidable collision occurs. A methodology and an architecture is presented in order to derive triggering decisions prior to straight V2V collisions. The introduced architecture represents a context-aware system that can sense, and react based on the vehicle environment. In the first step, the environment sensing is modeled by a state vector fusion for object target tracking. Then different models to predict a potential collisions based on the actual ego and object state are presented. Two physical models, the multi body model and the impulse and momentum theory, to estimate $C s$ in straight frontal V2V collisions are following. Afterwards it is investigated which exteroceptive sensor measurements can be used as input parameters to calculate these physical models. The relationships between frontal stiffness, mass and dimensions of different vehicles are analyzed based on four different data bases: NHTSA crash data base, vehicle blue prints, GIDAS database and V2V FEM crash simulations. It turns out that there is a good statistical relationship between the vehicles dimension and its mass and that there is no significant correlation between mass and mean frontal stiffness. The coefficient of restitution in $\mathrm{V} 2 \mathrm{~V}$ collisions shows a good correlation to vehicle collision speeds and the overlap. Due to the given uncertainties it is not feasible to calculate all possible accident scenarios shortly before collision in order to evaluate Cs. Hence, the data bases, a physical crash model and a statistical driver model are applied to build the Crash Severity Data Model (CSDM) that can evaluate a pre-crash scenario in a resource-saving manner. The CSDM defines a mapping of sensor data of the current vehicle environmental situation to a particular Cs and the necessary trigger strategy. The CSDM takes all of the possibilities of the collision outcome into account and is used as intelligence in the context-aware system. Through sampling, based on known probability density functions, a multidimensional feature space is created. Triggering thresholds are defined by hyperplanes in the resulting feature space. The contextaware system is evaluated with numerical simulation using 600 reconstructed GIDAS V2V accidents. Three different sensors are modeled to provide realistic pre-crash data. From the results it is concluded that the SVF architecture gives the best state estimates and that the CSDM selected right triggering strategy with relatively less uncertainty. The functionality has been demonstrated with an impulse and momentum model that deals with collisions with different overlaps and small relative angles between the vehicles longitudinal axis. To calculate $C s$, the impact momentum coefficient and an impact surface has to be predicted, that also could be extracted from FEM V2V simulations. However, $C s$ in small overlap frontal collisions is difficult to evaluate since it is inaccurate to predict if both vehicles are caught or slide during collision. To realize a more robust triggering decision, the percentile of the estimated crash severity PDF can be used instead of the expectation (for example the 30th percentile). Selecting the right trigger strategy remains a random process, especially when one considers the complex traffic environments anywhere in the world. In order to improve the collision prediction, advanced driver behavior detection methods (Al-Sultan et al., 2013) can be applied. It is also conceivable to enhance the Cs estimation using more information about the collision opponent to calculate more precise crash models. Technologies like the Ultra Wide Band (UWB) Radar for the predictive material inspection (Taylor, 2012) or Car2X-communication (Sommer and Dressler, 2014) can be considered to evaluate the collision opponents structure.

\section{References}

Al-Sultan, S., Al-Bayatti, A., Zedan, H., 2013. Context-aware driver behavior detection system in intelligent transportation systems. IEEE Tran. Veh. Technol. 62 (9), 4264-4275.

Altendorfer, R. 2009. Observable dynamics and coordinate systems for automotive target tracking. In: IEEE Intelligent Vehicles Symposium, pp. 741-746

Althoff, M., Stursberg, O., Buss, M., 2009. Model-based probabilistic collision detection in autonomous driving. IEEE Trans. Intell. Transp. Syst. 10, 299-310.

Andreas Meier, M.G., 2014. Precrash classification of car accidents for improved occupant safety systems. In: 2nd International Conference on System-Integrated Intelligence: Challenges for Product and Production Engineering, Procedia Technology, Vol. 15, pp. 198-207.

Appel, H., Krabbel, G., Vetter, D., 2002. Unfallforschung, Unfallmechanik und Unfallrekonstruktion. Springer-Verlag

Böhmländer, D., Yano, V., Brandmeier, T., Zimmer, A., Ling, L.L., Wong, C.-B. Dirndorfer, T., 2014. A novel approach for intelligent pre-crash threat assessment systems. In: IEEE 17th International Conference on Intelligent Transportation Systems (ITSC), pp. 954-961.

Bayless, S.H., Guan, A., Son, P., Murphy, S., Shaw, A.J., 2012. Trends in roadway domain active sensing. In: Connected Vehicle Insights, The Intelligent Transportation Society of America (ITS America)

Brach, R.M., Brach, R.M., 2011. Vehicle Accident Analysis and Reconstruction Methods, 2nd ed. SAE International.

Browne, A.L., Wood, F.D., Altan, O.D., Pre-collision assessment of potential collision severity for road vehicles, patent, US8321092 B2 (Nov. 2012).

Burg, H., 1982. Approximation von Trägheitsmomenten bei Personenkraftwagen, Vol. 20., pp. 61-62.

Chan, C.-Y., 2000. Fundamentals of Crash Sensing in Automotive Air Bag Systems. Soc. of Automotive Engineers, Warrendale, Pa.

Chan, C.-Y., 2002. On the detection of vehicular crashes-system characteristics and architecture. IEEE Trans. Veh. Technol. 51, 180-193.

Choi, H., Kim, Y., External airbag system for vehicles, patent, US7784817 B2 (2010).

Christiaans, T., 2004. Neoklassische Wachstumstheorie: Darstellung, Kritik und Erweiterung, 1st ed. Books on Demand, Norderstedt.

Chung, T.W., Kim, Y.S., Lee, S.H., Bae, J.H., Exterior airbag cushion for vehicle, patent, US8764062 B2 (Jul. 2014). 
Cleveland, W., Devlin, S., 1988. Locally-weighted regression: An approach to regression analysis by local fitting. J. Am. Stat. Assoc.

Continental AG, SRR20x Datasheet, http://www.conti-online.com/www/ download/industrial_sensors_de_de/themes/download/srr20x_datenblatt_de. pdf, (accessed 23.07.15).

Dirndorfer, T., Botsch, M., Knoll, A., 2011. Model-based analysis of sensor-noise in predictive passive safety algorithms. In: 11th Conference on ESV.

Eckert, A., Hartmann, B., Sevenich, M., Rieth, P., 2011. Emergency steer and brake assist - a systematic approach for system integration of two complementary driver assistance systems. In: 22th Conference on the ESV.

Eichberger, A., Wallner, D., Hirschberg, W., Cresnik, R., 2009. A situation based method to adapt the vehicle restraint system in frontal crashes to the accident scenario. In: Proceedings of the 21st International Technical Conference on the Enhanced Safety of Vehicles Conference (ESV) - International Congress Center Stuttgart, Germany.

Eidehall, A., Petersson, L., 2008. Statistical threat assessment for general road scenes using Monte Carlo sampling. IEEE Trans. Intell. Transp. Syst. 9, 137-147.

Elbel, M., (Ph. D. thesis) 2007. Kollisionsdynamik der beteiligten Fahrzeuge als Kausalitätskriterium für Beschleunigungsverletzungen der Halswirbelsäule. Medizinischen Fakultät der Universität Ulm.

Feser, M., McConnell, D., Brandmeier, T., Lauerer, C., 2006, April. Advanced crash discrimination using crash impact sound sensing (CISS). In: SAE Technical Paper 2006-01-1590, SAE International, Warrendale, PA.

Gstrein, G., Sinz, W., 2009. Improvement of airbag performance through pre-triggering. In: Eberle, W., Richert, J., Bullinger, W. (Eds.), International Technical Conference on the Enhanced Safety of Vehicles. Vehicle Safety Institute - Graz University of Technology/Daimler AG, Austria/Germany, Paper Number 09-0229.

Hakki, S., Hakki, A.-H., Collision air bag and flotation system, patent, US20080309060 A1 (Dec. 2008)

Huang, M., 2002. Vehicle Crash Mechanics. CRC Press Inc., Dearborn, Michigan, USA

Huibers, J., De Beer, E., 2001. Current front stiffness of European vehicles with regard to compatibility. In: Proceedings of the 17 th International Technical Conference on the ESV.

2015. Ibeo Automotive Systems GmbH, Datasheet ibeo LUX.

Jacobs, D.J., 2009. Best probability density function for random sampled data. Entropy 11, 1001-1024.

Jansson, J., (dissertations) 2005. Collision avoidance theory: with application to automotive collision mitigation, no. 950 in Linköping studies in science and technology. Univ, Linköping.

Kübler, L., Gargallo, S., Elsässer, K., 2008. Characterization and evaluation of frontal crash pulses with respect to occupant safety. In: 9th International Symposium and Exhibition on Sophisticated Car Occupant Safety Systems.

Kübler, L., Gargallo, S., Elsässer, K., 2009. Frontal crash pulse assessment with application to occupant safety. In: ATZ worldwide, Vol. 111, pp. 12-17.

Kalthoff, W., Becke, M., 2000. Die Stosszahl bei Auffahrkollisionen - Ein wesentlicher Parameter zur Bestimmung der HWS-Belastung., pp. 275-282.

Kalthoff, W., 2009. Die Stosszahl - ein entscheidener Parameter bei de Rekonstruktion eines Auffahrunfalls., pp. 375-379.

King, A.G., Vehicle collision severity estimation system, patent, US6917305 B2 (Jul. 2005).

Kwanghyun Cho, S.B.C., 2011. Design of an airbag deployment algorithm based on precrash information. IEEE Trans. Veh. Technol., 1438-1452.

Löffler, A., Quedenbaum, L., 2014. Crashsicherheit eines Technologietraegers für Elektrokleinstfahrzeuge. In: crash.tech, Munich.

Langwieder, K., 2013. Characteristics of Car Accidents Pre-Crash Phase. In: JSAE Annual Congress.

Lefévre, S., Vasquez, D., Laugier, C., 2014. A survey on motion prediction and risk assessment for intelligent vehicles. Robomech J. 1, 1-14.

Lefer, B., Rebolloso, I., (Master's thesis) 2012. Car-to-Truck Frontal Crash Compatibility, CHALMERS, Applied Mechanics.

Mühlfeld, F., Doric, I., Ertlmeier, R., Brandmeier, T., 2013. Statistical behavior modeling for driver-adaptive precrash systems. IEEE Trans. Intell. Transp. Syst. 14, 1764-1772.

McGehee, D.V., Carsten, O.M., 2010. Perception and biodynamics in unalerted precrash response. In: Annals of Advances in Automotive Medicine/Annual Scientific Conference, Vol. 54, pp. 315-332.

Metzger, J., Kübler, L., Gargallo, S., 2010. Characterization and evaluation of frontal crash pulses for USNCAP 2011. In: 10th International Symposium and Exhibition on Sophisticated Car Occupant Safety Systems.

Mishra, I., Airbag system, patent, US8172027 B2 (2012).

Monson, K.L., Germane, G.J., 1999, March. Determination and Mechanisms of Motor Vehicle Structural Restitution from Crash Test Data, SAE Technical Paper 1999-01-0097. SAE International, Warrendale, PA.
Naidu, V., Gopalratnam, G., 2010. Fusion architectures for 3d target tracking using radar and irst data. J. Aerosp. Sci. Technol. 62, 183-195.

NHTSA, Vehicle crash test database, http://www-nrd.nhtsa.dot.gov/database/veh/ veh.htm (accessed 24.05.16).

Nusholtz, G.S., Xu, L., Shi, Y., Domenico, L.D., 2005. Vehicle mass, stiffness and their relationship. In: The 19th International Technical Conference on the Enhanced Safety of Vehicles (ESV), Washington, DC, USA, RL http://www-nrd.nhtsa.dot. gov/pdf/esv/esv19/05-0413-O.pdf.

O'Brien, S., Ph. D. thesis 2010. Measurement and assessment of passenger vehicle compatibility in front and side collisions. School of Aerospace, Mechanical and Manufacturing Engineering, RMIT University.

Pedregosa, F., Varoquaux, G., Gramfort, A., Michel, V., Thirion, B., Grisel, O., Blondel, M., Prettenhofer, P., Weiss, R., Dubourg, V., Vanderplas, J., Passos, A., Cournapeau, D., Brucher, M., Perrot, M., Duchesnay, E., 2011. Scikit-learn: machine learning in python. J. Mach. Learn. Res. 12, 2825-2830.

Rölleke, M., Köhler, A., 2001. Smart Sensors for Passenger Safety Systems. http:// www.springerprofessional.de/smart-sensors-for-passenger-safety-systems/ 3433784.html, (visited on 16.03.15)

Rao, M.K., Strumoto, G.S., Prakah-Asante, K.O., Barbat, S.D., Schuster, P.J., Method for operating a pre-crash sensing system in a vehicle having external airbags, patent, US6950014 B2 (Sep. 2005).

Raymond Brach, M., Matthew Brach, R., 2007. Analysis of collisions: point mass mechanics and planar impact mechanics. Collis. Mag. 2.

Rong Li, X., Jilkov, V., 2003. Survey of maneuvering target tracking. part i. dynamic models. In: IEEE Transactions on Aerospace and Electronic Systems, Vol. 39, pp. 1333-1364.

Schöneburg, R., 1988. Zur Berechnung des Crashverhaltens von Fahrzeugen mit einfachen Strukturmodellen. VDI-Verlag.

Schimmelpfennig, K.H., Hebing, N., 1982. Geschwindigkeiten bei kreisförmiger Kurvenfahrt - Stabilitäts- und Sicherheitsgrenze. In: der Verkehrsunfall.

Schmitt, K.-U., Niederer, P.F., Cronin, D.S., Muser, M.H., Walz, F., 2007. Trauma Biomechanics: Accidental Injury in Traffic and Sports, 2nd ed. Springer, Berlin.

Schofield, B., (Ph. D. thesis) 2008. Model-based vehicle dynamics control for active safety. Department of Automatic Control, Lund Institute of Technology, Lund University.

Silverman, B.W., 1986. Density Estimation for Statistics and Data Analysis. CRC Press

Sommer, C., Dressler, F., 2014. Vehicular Networking. Cambridge University Press.

2013, July. Statistisches Bundesamt, Annual report on traffic accidents 2012, Tech. Rep. Fachserie 8 Reihe 7, Wiesbaden.

Swanson, J., Rockwell, T., Beuse, N., Summers, L., Summers, S., Park, B., 2003. Evaluation of stiffness measures from the US new car assessment program. In: 18th Conference on ESV.

Taylor, J.D., 2012. Ultrawideband Radar: Applications and Design. CRC Press.

Gennarelli, T.A., Wodzin, E., 2008. Abbreviated injury scale (AIS) 2005, update 2008. American Association for Automotive Medicine (AAAM).

Unselt, T., Unger, J., Krause, M., Hierlinger, T., 2015. The integrated safety concept of the ultra-compact electric vehicle visio. M. In: Proceedings of the 24th International Technical Conference on the Enhanced Safety of Vehicles (ESV) Gothenburg, Sweden.

Varat, M.S., 2003. Crash pulse modeling for vehicle safety research. In: International Technical Conference on the Enhanced Safety of Vehicles (ESV).

Velodyne, Datasheet Velodyne LiDAR PUCK VLP-16, http://velodyne.com/lidar/ web/pdf/doc/63-9229_VLP16_Datasheet_Rev-A_Web.pdf, (accessed 23.07.15).

Wallner, D., Eichberger, A., Hirschberg, W., 2009. A novel control algorithm for integration of active and passive vehicle safety systems in frontal collisions. J. Syst. Cybern. Inform. 8

Weber, Stefanie, (Ph. D. thesis) 2015. Standardreaktionen in kritischen Fahrsituationen - Studien mit dem Vehicle in the Loop zur Untersuchung des Reaktionsverhaltens bei Vorfahrtsmissachtung von rechts. Universität der Bundeswehr München.

Wegscheider, M., Prokop, G., 2005. Model-based comfort evaluation of driver assistance systems. In: 12. Erprobung und Simulation in der Fahrzeugentwicklung - Mess- und Versuchstechnik, Vol. 1900, VDI-Gesellschaft Fahrzeug- und Verkehrstechnik, Würzburg, Germany.

Wiacek, C.J., 1999. Driver/Vehicle Characteristics in Rear-End Precrash Scenarios Based on the General Estimates System (GES). In: International Congress and Exposition, SAE, Detroit, MI

Wohllebe, T., Gonter, M., Meinecke, M.-M., 2006. Potential of pre-crash restraints in frontal collisions. In: 8th International symposium, Sophisticated car occupant safety systems, Fraunhofer ICT, Karlsruhe, Germany.

World Health Organization, 2013. Global status report on road safety 2013: supporting a decade of action. World Health Organization (WHO), Geneva. 